\title{
Multinode Cooperative Communications in Wireless Networks
}

\author{
Ahmed K. Sadek, Student Member, IEEE, Weifeng Su, Member, IEEE, and K. J. Ray Liu, Fellow, IEEE
}

\begin{abstract}
In this paper, a class of cooperative communication protocols with arbitrary $N$-relay nodes is proposed for wireless networks, in which each relay coherently combines the signals received from $m(1 \leq m \leq N-1)$ previous relays in addition to the signal from the source. Exact symbol-error-rate (SER) expressions for an arbitrary $N$-node network employing $M$ 'ary phaseshift-keying (MPSK) modulation or quadrature-amplitude modulation (QAM) are provided for the proposed class of protocols. Further, approximate expressions for the SER are derived and shown to be tight at high enough signal-to-noise ratio (SNR). Our analysis reveals an interesting result: The class of cooperative protocols shares the same asymptotic performance at high enough SNR and does not depend on $m$, the number of previous nodes involving in coherent detection, hence, the asymptotic performance of a simple cooperative scenario in which each relay combines the signals from the source and the previous relay is exactly the same as that for a much more complicated scenario in which each relay combines the signals from the source and all the previous relays. The theoretical results also confirm that full diversity equal to the number of cooperating nodes is indeed achievable by the proposed protocols. Finally, we formulate a power-allocation problem in order to minimize the SER of the system. The analysis shows that the optimum power allocation at different nodes follows a certain ordering, and that the power-allocation scheme at high SNR does not depend on the channel quality of the direct link between the source and the destination. Closed-form solutions for the optimal power-allocation problem are provided for some network topologies. Simulation results confirm our theoretical analysis.
\end{abstract}

Index Terms-Cooperative diversity, multinode networks, optimal power allocation, performance analysis, virtual multiple-input-multiple-output (MIMO), wireless networks.

\section{INTRODUCTION}

$\mathbf{O}$ NE OF the major impairments to meeting the demands of next generation wireless networks for high data rate services is signal fading due to multipath propagation. To mitigate the fading effects, time, frequency, and spatial diversity techniques or a hybrid of them can be utilized. Among the different types of diversity, spatial diversity is of a special interest as it does not incur the system losses in terms of delay and bandwidth efficiency. Spatial diversity has been studied

Manuscript received March 19, 2005; revised April 3, 2006. The associate editor coordinating the review of this manuscript and approving it for publication was Dr. Sergio Barbarossa. This work was supported in part under Grant CTA-ARL DAAD 190120011.

A. K. Sadek and K. J. R. Liu are with the Department of Electrical and Computer Engineering, and the Institute for Systems Research, University of Maryland, College Park, MD 20742 USA (e-mail: aksadek@eng.umd.edu; kjrliu@eng.umd.edu).

W. Su is with the Department of Electrical Engineering, State University of New York, Buffalo, NY 14260 USA (e-mail: weifeng@eng.buffalo.edu).

Color version of Fig. 7 is available online at http://ieeexplore.ieee.org.

Digital Object Identifier 10.1109/TSP.2006.885773 intensively in the context of point-to-point communications, where it is introduced by utilizing the multiple-input-multiple-output (MIMO) systems, i.e., multiple antennas at the transmitter and/or the receiver sides [16]. It has been shown in the literature that utilizing MIMO systems can significantly increase the system throughput and reliability [1], [2]. On the other hand, in wireless networks, e.g., cellular and ad hoc networks, it might not be feasible to have multiple antennas installed at the terminals due to space limitations. To overcome this problem, and to benefit from the performance enhancement introduced by MIMO systems, the concept of cooperative diversity in wireless networks has been recently introduced [3]-[9], [15], [16]. In such a strategy, when a node has some information to transmit, it cooperates with other single-antenna terminals to transmit its information to a certain destination thus forming a virtual antenna array. The cooperating nodes act as the relay channels for the source node. Cooperative diversity techniques, or equivalently virtual MIMO systems, constitute a new communication paradigm where numerous questions need to be answered.

In [3] and [4], Laneman et al. proposed different cooperative diversity protocols and analyzed their performance in terms of outage behavior. The terms decode-and-forward and amplifyand-forward have been introduced in these two works. In decode-and-forward, each relay receives and decodes the signal transmitted by the source, and then it forwards the decoded signal to the destination which combines all of these copies in a proper way. Amplify-and-forward is a simpler technique, in which the relay amplifies the received signal and then forwards it to the destination. Although the noise is amplified along with the signal in this technique, we still gain spatial diversity by transmitting the signal over two spatially independent channels. Terminologies other than cooperative diversity are also used in the research community to refer to the same concept of achieving spatial diversity via forming virtual antennas. User cooperation diversity was introduced by Sendonaris et al. in [5] and [6]. In this two-part series of papers, the authors implemented a two-user code division multiple access (CDMA) cooperative system, where both users are active and use orthogonal codes to avoid multiple access interference. Another technique to achieve diversity that incorporates error-control-coding into cooperation is coded cooperation introduced by Hunter $e t$ al. in [10]. In [11], Boyer et al. introduced the concept of multihop diversity, in which each relay combines the signals received from all of the previous transmissions. This kind of spatial diversity is specially applicable in multihop ad hoc networks. The authors in [11] assumed that an error at any intermediate relay results in an error at the final destination, and through this assumption they derived upper bounds on the probability of outage and error 
performance of the system. These calculations were done for systems with binary phase-shift keying (BPSK) modulation.

In this paper, we propose a class of cooperative decode-and-forward protocols for arbitrary $N$-relay wireless networks, in which each relay can combine the signal received from the source along with one or more of the signals transmitted by previous relays. We consider selective relaying in which each receiving relay can judge on the quality of the receiving signal and decide whether to forward the received signal or not. This can be implemented in a practical system by allowing each relay to measure the received signal-to-noise ratio (SNR) and forward the signal if the SNR is higher than the prespecified threshold. Another possibility is to employ cyclic-redundancy-check encoding to data frames. In our proposed protocols, we refer to the scenario in which each relay combines the signals received from the previous $m$ relays along with that from the source as $\mathcal{C}(m)$, where $1 \leq m \leq N-1$. Note that the multihop diversity scheme introduced in [11] is similar to the scheme $\mathcal{C}(N-1)$ we are considering without selective relaying. First, we analyze the performance of a general cooperation scenario $\mathcal{C}(m), 1 \leq m \leq N-1$, and provide exact symbol-error-rate (SER) expressions for both $M$ 'ary phase-shift-keying (MPSK) and quadrature-amplitude modulation (QAM) signalling. Second, we derive an approximate expression for the SER of a general cooperation scenario that is shown to be tight at high enough SNR. Our theoretical analysis reveals a very interesting result: The class of proposed cooperative protocols $\{\mathcal{C}(m)\}_{m=1}^{N-1}$ shares the same asymptotic performance at high enough SNR. Hence, a simple cooperative protocol for a multinode network in which each relay combines the signals received from the source and the previous relay, namely $\mathcal{C}(1)$, has the same asymptotic performance as a much more complicated scenario, in which each relay combines the signals received from the source and all of the previous relays, namely $\mathcal{C}(N-1)$.

Such a result suggests using the simplest protocol $\mathcal{C}(1)$ as the cooperative strategy for a multinode decode-and-forward diversity network. The saving in the complexity in terms of channel-estimation computations when utilizing $\mathcal{C}(1)$ as a cooperative scenario is computed. Moreover, we compute the diversity gain of this cooperative scenario, and we show that it achieves full diversity gain equal to the number of cooperating terminals. Finally, we study optimal power allocation for the proposed class of cooperative diversity schemes, where the optimality is determined in terms of minimizing the SER of the system. We show that the optimal power allocation follows a certain ordering in which the source has allocated the largest amount of power and the first relay has the least power-allocation ratio. Also, the analysis reveals that at high enough SNR, the optimal power-allocation scheme does not depend on the quality of the direct link between the source and the destination. Closed-form solutions of optimal power allocation for some network topologies of practical interest are provided. The rest of the paper is organized as follows. In Section II, we present the system model and propose a class of cooperation protocols for multinode wireless networks. In Section III, we provide exact SER expressions for the class of cooperative protocols. In Section IV, we derive approximate SER expressions that are shown to be tight at enough high SNR, and we compute the diversity gain of each protocol. Finally, in Section V, we formulate the optimal power-allocation problem in order to minimize the SER performance of the system, and closed-form optimal power-allocation strategies are derived for some network topologies.

\section{System Model AND PROtocol Description}

We consider an arbitrary $N$-relay wireless network, where information is to be transmitted from a source to a destination. Due to the broadcast nature of the wireless channel, some relays can overhear the transmitted information and thus can cooperate with the source to send its data. The wireless link between any two nodes in the network is modeled as a Rayleigh fading narrowband channel with additive white Gaussian noise (AWGN). The channel fades for different links are assumed to be statistically independent. This is a reasonable assumption as the relays are usually spatially well separated. The additive noise at all receiving terminals is modeled as zero-mean complex Gaussian random variables with variance $\mathcal{N}_{o}$. For medium access, the relays are assumed to transmit over orthogonal channels, thus no interrelay interference is considered in the signal model.

The cooperation strategy we are considering employs a selective decode-and-forward protocol at the relaying nodes. Each relay can measure the received SNR and forwards the received signal if the SNR is higher than some threshold. For mathematical tractability of SER calculations, we assume the relays can judge whether the received symbols are decoded correctly or not and only forwards the signal if decoded correctly; otherwise, it remains idle. This assumption will be shown via simulations to be very close to the performance of the practical scenario of comparing the received SNR to a threshold, specially when the relays operate in a high SNR regime, as, for example, when the relays are selected close to the source node. The rationale behind this is that when the relays are closer to the source node, or more generally operate in a high SNR regime, the channel fading (outage event defined in [17]) becomes the dominant source of error [17], and hence measuring the received SNR gives a very good judgement on whether the received symbol can be decoded correctly or not with high probability.

Various scenarios for the cooperation among the relays can be implemented. A general cooperation scenario, denoted as $\mathcal{C}(m)$ $(1 \leq m \leq N-1)$, can be implemented in which each relay combines the signals received from the $m$ previous relays along with that received from the source. The simplest scenario $\mathcal{C}(1)$ among the class of proposed protocols is depicted in Fig. 1, in which each relay combines the signal received from the previous relay and the source. The most complicated scenario $\mathcal{C}(N-1)$ is depicted in Fig. 2, in which each relay combines the signals received from all of the previous relays along with that from the source, and thus is similar to the scenario considered in [11]. This is the most sophisticated scenario and should provide the best performance in the class of proposed protocols $\{\mathcal{C}(m)\}_{m=1}^{N-1}$ as in this case each relay utilizes the information from all previous phases of the protocol. In all of the considered cooperation scenarios, the destination coherently combines the signals received from the source and all of the relays. In the sequel, we 


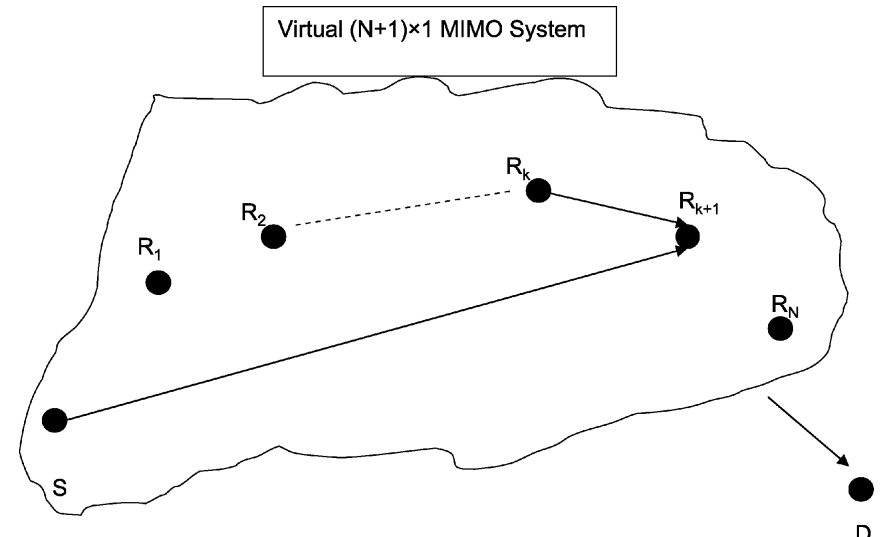

Fig. 1. Illustrating cooperation under $\mathcal{C}(1)$ : The $(k+1)$ th relay combines the signals received from the source and the $k$ th relay.

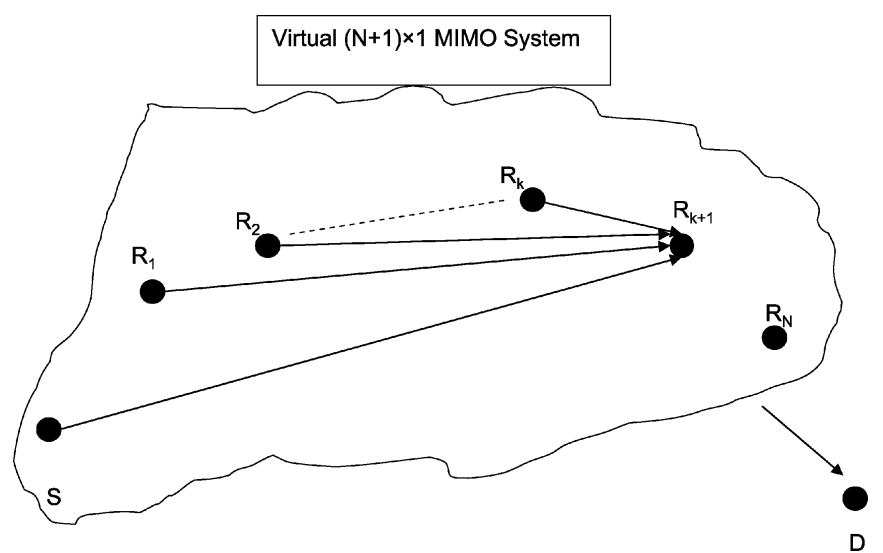

Fig. 2. Illustrating cooperation under $\mathcal{C}(N-1)$ : The $(k+1)$ th relay combines the signals received from the source and all of the previous relays.

focus on presenting the system model for a general cooperative scheme $\mathcal{C}(m)$ for any $1 \leq m \leq N-1$.

For a general scheme $\overline{\mathcal{C}}(m), 1 \leq m \leq N-1$, each relay decodes the information after combining the signals received from the source and the previous $m$ relays. The cooperation protocol has $(N+1)$ phases. In phase 1 , the source transmits the information, and the received signal at the destination and the $i$ th relay can be modeled, respectively, as

$$
\begin{aligned}
y_{s, d} & =\sqrt{P_{0}} h_{s, d} x+n_{s, d} \\
y_{s, r_{i}} & =\sqrt{P_{0}} h_{s, r_{i}} x+n_{s, r_{i}}, \quad 1 \leq i \leq N
\end{aligned}
$$

where $P_{0}$ is the power transmitted at the source, $x$ is the transmitted symbol with unit power, $h_{s, d} \sim C N\left(0, \sigma_{s, d}^{2}\right)$ and $h_{s, r_{i}} \sim C N\left(0, \sigma_{s, r_{i}}^{2}\right)$ are the channel fading coefficients between the source and the destination, and $i$ th relay, respectively, and $C N\left(\alpha, \sigma^{2}\right)$ denotes a circularly symmetric complex Gaussian random variable with mean $\alpha$ and variance $\sigma^{2}$. The terms $n_{s, d}$ and $n_{s, r_{i}}$ denote the AWGN. In phase 2, if the first relay correctly decodes, it forwards the decoded symbol with power $P_{1}$ to the destination; otherwise, it remains idle.
Generally in phase $l, 2 \leq l \leq N$, the $l$ th relay combines the received signals from the source and the previous $\min \{m, l-1\}$ relays using a maximal-ratio-combiner (MRC) as follows:

$$
y_{r_{l}}=\sqrt{P_{0}} h_{s, r_{l}}^{*} y_{s, r_{l}}+\sum_{i=\max (1, l-m)}^{l-1} \sqrt{\hat{P}_{i}} h_{r_{i}, r_{l}}^{*} y_{r_{i}, r_{l}}
$$

where $h_{r_{i}, r_{l}} \sim C N\left(0, \sigma_{r_{i}, r_{l}}^{2}\right)$ is the channel fading coefficient between the $i$ th and the $l$ th relays. In (2), $y_{r_{i}, r_{l}}$ denotes the signal received at the $l$ th relay from the $i$ th relay, and can be modeled as

$$
y_{r_{i}, r_{l}}=\sqrt{\hat{P}_{i}} h_{r_{i}, r_{l}} x+n_{r_{i}, r_{l}}
$$

where $\hat{P}_{i}$ is the power transmitted at relay $i$ in phase $(i+1)$, and $\hat{P}_{i}=P_{i}$ if relay $i$ correctly decodes the transmitted symbol; otherwise, $\hat{P}_{i}=0$. The $l$ th relay uses $y_{r_{l}}$ in (2) as the detection statistics. If relay $l$ decodes correctly, it transmits with power $\hat{P}_{l}=P_{l}$ in phase $(l+1)$; otherwise, it remains idle. Finally, in phase $(N+1)$, the destination coherently combines all of the received signals using an MRC as follows:

$$
y_{d}=\sqrt{P_{0}} h_{s, d}^{*} y_{s, d}+\sum_{i=1}^{N} \sqrt{\hat{P}_{i}} h_{r_{i}, d}^{*} y_{r_{i}, d} .
$$

In all the cooperation scenarios considered, the total transmitted power is fixed as $P_{0}+\sum_{i=1}^{N} P_{i}=P$.

\section{EXACt SER PERformance ANALYsis}

In this section, we present SER performance analysis for a general cooperative scheme $\mathcal{C}(m)$ for any $1 \leq m \leq N-1$. Exact SER expressions of this general scheme is provided for systems utilizing either MPSK or multi-QAM (MQAM) modulation.

First, we introduce some terminologies that will be used throughout the paper. For a given transmission, each relay can be in one of two states: Either it decoded correctly or not. Let us define a $1 \times n, 1 \leq n \leq N$, vector $\mathbf{S}_{n}$ to represent the states of the first $n$ relays for a given transmission. The $k$ th entry of the vector $\mathbf{S}_{n}$ denotes the state of the $k$ th relay as follows:

$S_{n}[k]=\left\{\begin{array}{ll}1, & \text { if relay } k \text { correctly decodes, } \\ 0, & \text { otherwise, }\end{array} \quad 1 \leq k \leq n\right.$.

Since the decimal value of the binary vector $\mathbf{S}_{n}$ can take on values from 0 to $2^{n}-1$, for convenience, we denote the state of the network by an integer decimal number. Let $\mathbf{B}_{x, n}=$ $\left(B_{x, n}[1], B_{x, n}[2], \ldots, B_{x, n}[n]\right)$ be the $1 \times n$ binary representation of a decimal number $x$, with $B_{x, n}$ [1] being the most significant bit. So, $\mathbf{S}_{N}=\mathbf{B}_{x, N}$ indicates that the $k$ th relay, $1 \leq k \leq$ $N$, is in state $S_{N}[k]=B_{x, N}[k]$.

\section{A. Exact SER for General Cooperation Scheme}

We consider a general cooperation scheme $\mathcal{C}(m), 1 \leq m \leq$ $N-1$, in which the $k$ th $(1 \leq k \leq N)$ relay coherently combines the signals received from the source along with the signals received from the previous $\min \{m, k-1\}$ relays. The state of each relay in this scheme depends on the states of the previous $m$ 
relays, i.e., whether these relays decoded correctly or not. This is due to the fact that the number of signals received at each relay depends on the number of relays that decoded correctly from the previous $m$ relays. Hence, the joint probability of the states is given by

$$
\begin{aligned}
P\left(\mathbf{S}_{N}\right)= & P\left(S_{N}[1]\right) P\left(S_{N}[2] \mid S_{N}[1]\right) \\
& \ldots P\left(S_{N}[N] \mid S_{N}[N-1], \ldots, S_{N}[N-m]\right) .
\end{aligned}
$$

Conditioning on the network state, which can take $2^{N}$ values, the probability of error at the destination given the channel state information (CSI) can be calculated using the law of total probability as follows:

$$
P_{e \mid \mathrm{CSI}}=\sum_{i=0}^{2^{N}-1} \operatorname{Pr}\left(e \mid \mathbf{S}_{N}=\mathbf{B}_{i, N}\right) \operatorname{Pr}\left(\mathbf{S}_{N}=\mathbf{B}_{i, N}\right)
$$

where $e$ denotes the event that the destination decoded in error. The summation in (7) is over all possible states of the network.

Now, let us compute the terms in (7). The destination collects the copies of the signal transmitted in the previous phases using an MRC (4). The resulting SNR at the destination can be computed as

$$
\mathrm{SNR}_{d}=\frac{P_{0}\left|h_{s, d}\right|^{2}+\sum_{j=1}^{N} P_{j} B_{i, N}[j]\left|h_{r_{j}, d}\right|^{2}}{\mathcal{N}_{o}}
$$

where $B_{i, N}[j]$ takes value 1 or 0 and determines whether the $j$ th relay has decoded correctly or not. The $k$ th relay coherently combines the signals received from the source and the previous $m$ relays. The resulting SNR can be calculated as

$$
\mathrm{SNR}_{r_{k}}^{m}=\frac{P_{0}\left|h_{s, r_{k}}\right|^{2}+\sum_{j=\max (1, k-m)}^{k-1} P_{j} B_{i, N}[j]\left|h_{r_{j}, r_{k}}\right|^{2}}{\mathcal{N}_{o}} .
$$

If MPSK modulation is used in the system, with instantaneous SNR $\gamma$, the SER given the channel state information is given by [12]

$$
P_{\mathrm{CSI}}^{\mathrm{PSK}}=\Psi_{\mathrm{PSK}}(\gamma) \triangleq \frac{1}{\pi} \int_{0}^{(M-1) \pi / M} \exp \left(-\frac{b_{\mathrm{PSK}} \gamma}{\sin ^{2}(\theta)}\right) d \theta
$$

where $b_{\mathrm{PSK}}=\sin ^{2}(\pi / M)$. If MQAM $\left(M=2^{k}\right.$ with $k$ even $)$ modulation is used in the system, the corresponding conditional SER can be expressed as [12]

$$
P_{\mathrm{CSI}}^{\mathrm{QAM}}=\Psi_{\mathrm{QAM}}(\gamma) \triangleq 4 C Q\left(\sqrt{b_{\mathrm{QAM}} \gamma}\right)-4 C^{2} Q^{2}\left(\sqrt{b_{\mathrm{QAM}}}\right)
$$

in which $C=1-1 / \sqrt{M}, b_{\mathrm{QAM}}=3 /(M-1)$, and $Q(x)$ is the complementary distribution function (CDF) of the Gaussian distribution, and is defined as $Q(x)=(1 / \sqrt{2 \pi}) \int_{x}^{\infty} \exp \left(-t^{2} / 2\right) d t$.

Let us focus on computing the SER in the case of MPSK modulation, and the same procedure is applicable for the case of MQAM modulation. From (8), and for a given network state
$\mathbf{S}_{N}=\mathbf{B}_{i, N}$, the conditional SER at the destination can be computed as

$$
\operatorname{Pr}\left(e \mid \mathbf{S}_{N}=\mathbf{B}_{i, N}\right)=\Psi_{\mathrm{PSK}}\left(\mathrm{SNR}_{d}\right) .
$$

Denote the conditional probability that the $k$ th relay is in state $B_{i, N}[k]$ given the states of the previous $m$ relays by $P_{k, i}^{m}$. From (9), this probability can be computed as follows:

$$
\begin{aligned}
P_{k, i}^{m} & \triangleq \operatorname{Pr}\left(S_{N}[k]=B_{i, N}[k] \mid S_{N}[k-1]\right. \\
& \left.=B_{i, N}[k-1], \ldots, S_{N}[k-m]=B_{i, N}[k-m]\right) \\
& = \begin{cases}\Psi_{\mathrm{PSK}}\left(\mathrm{SNR}_{r_{k}}^{m}\right), & \text { if } B_{i, N}[k]=0 \\
1-\Psi_{\mathrm{PSK}}\left(\operatorname{SNR}_{r_{k}}^{m}\right), & \text { if } B_{i, N}[k]=1 .\end{cases}
\end{aligned}
$$

To compute the average SER, we need to average the probability in (7) over all channel realizations, i.e., $P_{\mathrm{SER}}(m)=$ $E_{\mathrm{CSI}}\left[P_{e \mid \mathrm{CSI}}\right]$. Using (6), (12), and (13), $P_{\mathrm{SER}}(m)$ can be expanded as follows:

$$
P_{\mathrm{SER}}(m)=\sum_{i=0}^{2^{N}-1} E_{\mathrm{CSI}}\left[\Psi_{\mathrm{PSK}}\left(\mathrm{SNR}_{d}\right) \prod_{k=1}^{N} P_{k, i}^{m}\right] .
$$

Since the channel fades between different pairs of nodes in the network are statistically independent by the virtue that different nodes are not colocated, the quantities inside the expectation operator in (14) are functions of independent random variables, and thus can be further decomposed as

$$
P_{\mathrm{SER}}(m)=\sum_{i=0}^{2^{N}-1}\left\{E_{\mathrm{CSI}}\left[\Psi_{\mathrm{PSK}}\left(\mathrm{SNR}_{d}\right)\right] \prod_{k=1}^{N} E_{\mathrm{CSI}}\left[P_{k, i}^{m}\right]\right\} .
$$

The above analysis is applicable to the MQAM case by changing the function $\Psi_{\mathrm{PSK}}(\cdot)$ into $\Psi_{\mathrm{QAM}}(\cdot)$.

Since the channels between the nodes are modeled as Rayleigh fading channels, the absolute norm square of any channel realization $h_{i, j}$ between any two nodes $i$ and $j$ in the network has an exponential distribution with mean $\sigma_{i, j}^{2}$. Hence, $E_{\mathrm{CSI}}\left[\Psi_{q}(\gamma)\right]$ can be expressed as

$$
E_{\mathrm{CSI}}\left[\Psi_{q}(\gamma)\right]=\int_{\gamma} \Psi_{q}(\gamma) f(\gamma) d \gamma
$$

where $f(\gamma)$ is the probability density function of the random variable $\gamma$, and $q=1(q=2)$ correspond to MPSK (MQAM), respectively. If $\gamma$ is an exponentially distributed random variable with mean $\bar{\gamma}$, then it can be shown [12] that $E_{\mathrm{CSI}}\left[\Psi_{q}(\gamma)\right]$ is given by

$$
E_{\mathrm{CSI}}\left[\Psi_{q}(\gamma)\right]=F_{q}\left(1+\frac{b_{q} \bar{\gamma}}{\sin ^{2}(\theta)}\right)
$$

where $F_{q}(\cdot)$ and the constant $b_{q}$ are defined as

$$
\begin{aligned}
F_{1}(x(\theta)) & =\frac{1}{\pi} \int_{0}^{(M-1) \pi / M} \frac{1}{x(\theta)} d \theta \\
b_{1} & =b_{\mathrm{PSK}} \\
F_{2}(x(\theta)) & =\frac{4 C}{\pi} \int_{0}^{\pi / 2} \frac{1}{x(\theta)} d \theta-\frac{4 C^{2}}{\pi} \int_{0}^{\pi / 4} \frac{1}{x(\theta)} d \theta \\
b_{2} & =\frac{b_{\mathrm{QAM}}}{2}
\end{aligned}
$$


In order to get the previous expressions, we use two special properties of the $Q(\cdot)$ function, specifically, $Q(x)=(1 / \pi) \int_{0}^{\pi / 2} \exp \left(-\left(x^{2} / 2 \sin ^{2}(\theta)\right)\right) d \theta$, and $Q^{2}(x)=$ $(1 / \pi) \int_{0}^{\pi / 4} \exp \left(-\left(x^{2} / 2 \sin ^{2}(\theta)\right)\right) d \theta$ for $x \geq 0$ [12], [13].

Averaging over all the Rayleigh fading channel realizations, the SER at the destination for a given network state $\mathbf{B}_{i, N}$ is given by

$$
\begin{aligned}
& E_{\mathrm{CSI}}\left(\Psi_{q}\left(\mathrm{SNR}_{d}\right)\right) \\
& \quad=F_{q}\left[\left(1+\frac{b_{q} P_{0} \sigma_{s, d}^{2}}{\mathcal{N}_{o} \sin ^{2}(\theta)}\right) \prod_{j=1}^{N}\left(1+\frac{b_{q} B_{i, N}[j] P_{j} \sigma_{r_{j}, d}^{2}}{\mathcal{N}_{o} \sin ^{2}(\theta)}\right)\right] .
\end{aligned}
$$

Similarly, the probability that the $k$ th relay is in state $B_{i, N}[k]$ given the states of the previous $m$ relays is given by

$$
E_{\mathrm{CSI}}\left[P_{k, i}^{m}\right]=G_{k}^{m}\left(B_{i, N}[k]\right)
$$

where $G_{k}^{m}(\cdot)$ is defined as (21), shown at the bottom of the page, in which $F_{q}(\cdot)$ and the constant $b_{q}$ are specified in (18). As a summary, the SER in (15) of the cooperative multinode system employing scenario $\mathcal{C}(m)$ with MPSK or MQAM modulation can be determined from (19)-(21) in Theorem 1.

Theorem 1: The SER of an $N$-relay decode-and-forward cooperative diversity network utilizing protocol $\mathcal{C}(m), 1 \leq m \leq$ $N-1$, and MPSK or MQAM modulation is given by (22), as shown at the bottom of the page, where the functions $F_{q}(\cdot)$ and $G_{k}^{m}(\cdot)$ are defined in (18) and (21), respectively.

\section{B. Verifying the Validity of Our Theoretical Model for Selective Relaying}

In this section, we will illustrate with some simulation experiments the validity of the theoretical results we obtained. In the simulations, we considered only cooperative protocol $\mathcal{C}(1)$. The number of relays is taken to be $N=1,2,3$, in addition to the source and the destination nodes. We considered two simulation setups. In the first setup, we simulate the SER performance under the assumption that the relay correctly judges whether the received signal is decoded correctly or not, i.e., no error propagation, which is the model analyzed in the paper. In the second

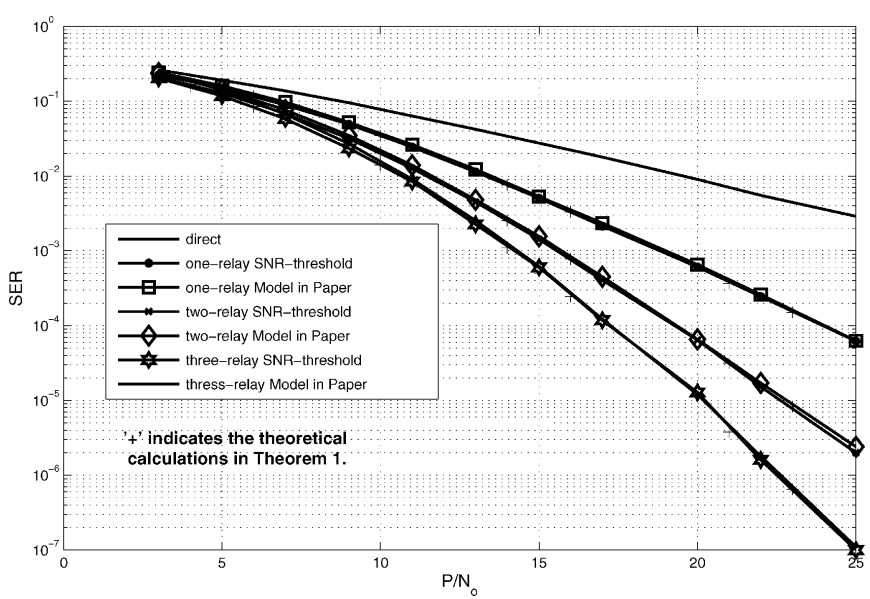

Fig. 3. SER versus SNR for two different scenarios. The first is the simulated SER for the model described in the paper in which the relays know whether each symbol is decoded correctly or not. The second is the simulated SER for a practical scenario in which the relay forwards the decoded symbol based on comparing the received SNR with a threshold. Also, the exact SER expression in (22) is plotted as "+." The cooperation protocol utilized is $\mathcal{C}(1)$ and the modulation scheme is QPSK.

setup, we consider the more practical scenario in which each relay compares the instantaneous received SNR to a threshold and, hence, decides whether to forward the received signal or not, and, thus, error propagation is allowed (the threshold is taken equal to $3 \mathrm{~dB}$ here and is selected by experiment). The relays are considered closer to the source than the destination. The channel variance depends on the distance $l$ and propagation path loss $\alpha$ as follows $\sigma^{2} \propto l^{-\alpha}$, and $\alpha=3$ in our simulations. The channel gains are as follows: $\sigma_{s, r_{i}}^{2}=8 \sigma_{s, d}^{2}$, and $\sigma_{r_{i}, d}^{2}=\sigma_{s, d}^{2}$. The noise variance is taken to be $N_{o}=1$. The total transmitted power in each case is considered fixed to $P$.

Fig. 3 depicts the SER versus $P / N_{o}$ performance of cooperation scenario $\mathcal{C}(1)$ with quadrature phase-shift keying (QPSK). As shown in the figure, the performance curves of the two previously described simulation setups are very close for different number of relays. This validates that our model in the paper for selective relaying assumed for mathematical tractability has close performance to that of practical selective relaying when

$$
G_{k}^{m}(x)= \begin{cases}F_{q}\left[\left(1+\frac{b_{q} P_{0} \sigma_{s, r_{k}}^{2}}{\mathcal{N}_{o} \sin ^{2}(\theta)}\right) \prod_{j=\max (1, k-m)}^{k-1}\left(1+\frac{b_{q} B_{i, N}[j] P_{j} \sigma_{r_{j}, r_{k}}^{2}}{\mathcal{N}_{o} \sin ^{2}(\theta)}\right)\right], & \text { if } x=0 \\ 1-F_{q}\left[\left(1+\frac{b_{q} P_{0} \sigma_{s, r_{k}}^{2}}{\mathcal{N}_{o} \sin ^{2}(\theta)}\right) \prod_{j=\max (1, k-m)}^{k-1}\left(1+\frac{b_{q} B_{i, N}[j] P_{j} \sigma_{r_{j}, r_{k}}^{2}}{\mathcal{N}_{o} \sin ^{2}(\theta)}\right)\right], & \text { if } x=1\end{cases}
$$

$$
P_{\mathrm{SER}}(m)=\sum_{i=0}^{2^{N}-1} F_{q}\left[\left(1+\frac{b_{q} P_{0} \sigma_{s, d}^{2}}{\mathcal{N}_{o} \sin ^{2}(\theta)}\right) \prod_{j=1}^{N}\left(1+\frac{b_{q} B_{i, N}[j] P_{j} \sigma_{r_{j}, d}^{2}}{\mathcal{N}_{o} \sin ^{2}(\theta)}\right)\right] \prod_{k=1}^{N} G_{k}^{m}\left(B_{i, N}[k]\right)
$$


comparing the SNR to a threshold. The intuition behind this is, as we illustrated before, that when the relays in general operate in a high SNR regime, in this case the relays are closer to the source node, the error propagation from the relays becomes negligible, and this is due to the fact that the channel outage event (SNR less than the threshold) becomes the dominating error event as proved in [17].

The performance of direct transmission without any relaying is also shown in Fig. 3 as a benchmark for a no-diversity scheme. Moreover, the exact SER expression from Theorem 1 is depicted as a "+" mark. It is clear from the depicted figure that the analytical SER expression in $(22)$ for scenario $\mathcal{C}(1)$ exactly matches the simulation results for each case. This confirms our theoretical analysis. The results also reveal that the cooperative diversity protocols can achieve full diversity gain in the number of cooperating terminals, which can be seen from the slopes of the performance curves which become more steeper with increasing the number of relays.

\section{APPROXIMATE SER EXPRESSION}

In Section III, we provided exact expressions for the SER of a general cooperative scheme $\mathcal{C}(m), 1 \leq m \leq N-1$, for arbitrary $N$-relay networks with either MPSK or MQAM modulation. The derived SER expressions, however, involve $2^{N}$ terms and integral functions. In this section, we provide approximate expressions for the SER performance of the proposed class of cooperative diversity schemes. The approximation is derived at high SNR and yields simple expressions that can provide insights to understanding the factors affecting the system performance, which helps in designing different network functions as power allocation, scheduling, routing, and node selection.

\section{A. SER Approximation for General Cooperative Protocol}

One can see that any term in the exact SER formulation (22) in Theorem 1 consists of the product of two quantities.

1) One of them is

$$
F_{q}\left[\left(1+\frac{b_{q} P_{0} \sigma_{s, d}^{2}}{\mathcal{N}_{o} \sin ^{2}(\theta)}\right) \prod_{j=1}^{N}\left(1+\frac{b_{q} B_{i, N}[j] P_{j} \sigma_{r_{j}, d}^{2}}{\mathcal{N}_{o} \sin ^{2}(\theta)}\right)\right]
$$

which corresponds to the conditional SER at the destination for a given network state $\mathbf{B}_{i, N}$.
2) The other one is the probability of the network being in that state, and is given by $\prod_{k=1}^{N} G_{k}^{m}\left(B_{i, N}[k]\right)$.

At high enough SNR, the probability of error $F_{q}(\cdot)$ is sufficiently small compared to 1 , thus, we can assume that $1-F_{q}(\cdot) \simeq 1$. Hence, the only terms in the second quantity $\prod_{k=1}^{N} G_{k}^{m}\left(B_{i, N}[k]\right)$ that will count are those corresponding to relays that have decoded in error. For convenience, we make the following definition: Let $\Omega_{i}(n, m)$ denote the subset of nodes that decode correctly from node $\max (1, n-m)$ till node $n-1$, when the network was in state $\mathbf{B}_{i, N}$. More specifically

$$
\begin{aligned}
& \Omega_{i}(n, m) \\
& \triangleq\left\{\text { relay } j \text { : s.t. } B_{i, N}[j]=1, \max (1, n-m) \leq j \leq n-1 .\right\} .
\end{aligned}
$$

Then, the SER formulation (22) in Theorem 1 can be approximated as (24), shown at the bottom of the page, where $\Omega^{c}$ is the complementary set of $\Omega$, i.e., the set of nodes that decoded erroneously.

First, we simplify the first term corresponding to the SER at the destination. Using the definition of $F_{q}$ in (18), and ignoring all the $1 \mathrm{~s}^{1}$ in $F_{q}(\cdot)$ in $(24)$, the conditional SER at the destination for a given network state $\mathbf{B}_{i, N}$ can be approximated as

$$
\begin{array}{r}
F_{q}\left[\left(1+\frac{b_{q} P_{0} \sigma_{s, d}^{2}}{\mathcal{N}_{o} \sin ^{2}(\theta)}\right) \prod_{j=1}^{N}\left(1+\frac{b_{q} B_{i, N}[j] P_{j} \sigma_{r_{j}, d}^{2}}{\mathcal{N}_{o} \sin ^{2}(\theta)}\right)\right] \\
\simeq \frac{\mathcal{N}_{o}^{1+\left|\Omega_{i}(N+1, N)\right|} g_{q}\left(1+\left|\Omega_{i}(N+1, N)\right|\right)}{b_{q}^{1+\left|\Omega_{i}(N+1, N)\right|} P_{0} \sigma_{s, d}^{2} \prod_{j \in \Omega_{i}(N+1, N)} P_{j} \sigma_{r_{j}, d}^{2}}
\end{array}
$$

where $\left|\Omega_{i}(N+1, N)\right|$ denotes the cardinality of the set $\Omega_{i}(N+$ $1, N)$, i.e., the number of nodes that decode correctly, which also denotes the number of signal copies transmitted from the $N$ relays to the destination at network state $\mathbf{B}_{i, N}$. The function $g_{q}(\cdot)$ in (25) is specified as (26), shown at the bottom of the page.

Let us write the transmitter powers allocated at the source and different relays as a ratio of the total available power $P$ as

${ }^{1}$ The tightness of these approximations can be proved easily by computing some limit functions for $F_{q}(x)$ and $1-F_{q}(x)$ as $x$, which denotes an affine function of the power and goes to $\infty$. For page limitations, we only include the proof for the single-relay scenario using MPSK in the Appendix.

$$
P_{\mathrm{SER}}(m) \simeq \sum_{i=0}^{2^{N}-1} F_{q}\left[\left(1+\frac{b_{q} P_{0} \sigma_{s, d}^{2}}{\mathcal{N}_{o} \sin ^{2}(\theta)}\right) \prod_{j=1}^{N}\left(1+\frac{b_{q} B_{i, N}[j] P_{j} \sigma_{r_{j}, d}^{2}}{\mathcal{N}_{o} \sin ^{2}(\theta)}\right)\right] \prod_{k \in \Omega^{c}(N+1, N)} G_{k}^{m}\left(B_{i, N}[k]\right)
$$

$$
g_{q}(x)= \begin{cases}\frac{1}{\pi} \int_{0}^{(M-1) \pi / M} \sin ^{2 x}(\theta) d \theta, & \text { for MPSK, } q=1 \\ \frac{4 C}{\pi}\left[\int_{0}^{\pi / 2} \sin ^{2 x}(\theta) d \theta-C \int_{0}^{\pi / 4} \sin ^{2 x}(\theta) d \theta\right], & \text { for MQAM, } q=2\end{cases}
$$


follows, $P_{0}=a_{0} P$, and $P_{i}=a_{i} P, 1 \leq i \leq N$, in which the power ratios are normalized as $a_{0}+\sum_{i=1}^{N} a_{i}=1$. One can then rewrite (25) in terms of the power-allocation ratios as follows:

$$
\begin{gathered}
F_{q}\left[\left(1+\frac{b_{q} P_{0} \sigma_{s, d}^{2}}{\mathcal{N}_{o} \sin ^{2}(\theta)}\right) \prod_{j=1}^{N}\left(1+\frac{b_{q} B_{i, N}[j] P_{j} \sigma_{r_{j}, d}^{2}}{\mathcal{N}_{o} \sin ^{2}(\theta)}\right)\right] \\
\simeq \frac{\left(\frac{\mathcal{N}_{o}}{P}\right)^{1+\left|\Omega_{i}(N+1, N)\right|} g_{q}\left(1+\left|\Omega_{i}(N+1, N)\right|\right)}{b_{q}^{1+\left|\Omega_{i}(N+1, N)\right|} a_{0} \sigma_{s, d}^{2} \prod_{j \in \Omega_{i}(N+1, N)} a_{j} \sigma_{r_{j}, d}^{2}}
\end{gathered}
$$

Note that the SNR term $\left(\mathcal{N}_{o} / P\right)$ in $(27)$ is of order $\left(1+\mid \Omega_{i}(N+\right.$ $1, N) \mid$ ). This is intuitively meaningful since the destination receives $\left(1+\left|\Omega_{i}(N+1, N)\right|\right)$ copies of the signal, in which the term 1 is due to the copy from the source. Thus, (27) decays as $\mathrm{SNR}^{-\left(1+\left|\Omega_{i}(N+1, N)\right|\right)}$ at high SNR.

At the $k$ th relay, $1 \leq k \leq N$, the conditional SER for a given network state $\mathbf{B}_{i, N}$ can be similarly approximated as

$$
\begin{gathered}
F_{q}\left[\left(1+\frac{b_{q} P_{0} \sigma_{s, r_{k}}^{2}}{\mathcal{N}_{o} \sin ^{2}(\theta)}\right) \prod_{j=\max (1, k-m)}^{k-1}\left(1+\frac{b_{q} B_{i, N}[j] P_{j} \sigma_{r_{j}, r_{k}}^{2}}{\mathcal{N}_{o} \sin ^{2}(\theta)}\right)\right] \\
\simeq \frac{\left(\frac{\mathcal{N}_{o}}{P}\right)^{1+\left|\Omega_{i}(k, m)\right|} g_{q}\left(1+\left|\Omega_{i}(k, m)\right|\right)}{b_{q}^{1+\left|\Omega_{i}(k, m)\right|} a_{0} \sigma_{s, r_{k}}^{2} \prod_{j \in \Omega_{i}(k, m)} a_{j} \sigma_{r_{j}, r_{k}}^{2}}
\end{gathered}
$$

where $\left.\left|\Omega_{i}(k, m)\right|\right)$ is the number of relays that decodes correctly from the previous $\min (k-1, m)$ relays. The SNR in (28) is of order $1+\left|\Omega_{i}(k, m)\right|$. From (28), the product $\prod_{k \in \Omega_{i}^{c}(N+1, N)} G_{k}^{m}\left(B_{i, N}[k]\right)$ in (24) is given by

$$
\begin{aligned}
& \prod_{k \in \Omega_{i}^{c}(N+1, N)} G_{k}^{m}\left(B_{i, N}[k]\right) \\
= & \prod_{k \in \Omega_{i}^{c}(N+1, N)} \frac{\left(\frac{\mathcal{N}_{o}}{P}\right)^{1+\left|\Omega_{i}(k, m)\right|} g_{q}\left(1+\left|\Omega_{i}(k, m)\right|\right)}{b_{q}^{1+\left|\Omega_{i}(k, m)\right|} a_{0} \sigma_{s, r_{k}}^{2} \prod_{j \in \Omega_{i}(k, m)} a_{j} \sigma_{r_{j}, r_{k}}^{2}}
\end{aligned}
$$

in which the SNR is of order

$$
\begin{aligned}
\sum_{k \in \Omega_{i}^{c}(N+1, N)}\left(1+\left|\Omega_{i}(k, m)\right|\right)= & \left|\Omega_{i}^{c}(N+1, N)\right| \\
& +\sum_{k \in \Omega_{i}^{c}(N+1, N)}\left|\Omega_{i}(k, m)\right| .
\end{aligned}
$$

Substituting (27) and (29) into (24), we get (30), as shown at the bottom of the page, where

$$
\begin{aligned}
d_{i}=1+\left|\Omega_{i}(N+1, N)\right|+\mid & \Omega_{i}^{c}(N+1, N) \mid \\
& +\sum_{k \in \Omega_{i}^{c}(N+1, N)}\left|\Omega_{i}(k, m)\right| .
\end{aligned}
$$

From (30), we can see that the SNR is of order $d_{i}$. Since $\left|\Omega_{i}(N+1, N)\right|+\left|\Omega_{i}^{c}(N+1, N)\right|=N$, the order $d_{i}$ can be lower bounded as follows:

$$
d_{i}=1+N+\sum_{k \in \Omega_{i}^{c}(N+1, N)}\left|\Omega_{i}(k, m)\right| \geq N+1
$$

in which the equality holds if and only if $\sum_{k \in \Omega^{c}}(N+1, N)$ $\left|\Omega_{i}(k, m)\right|=0$. Thus, the smallest order of the SNR is $N+1$.

The equality in (31) holds if and only if $\left|\Omega_{i}(k, m)\right|=0$, for any $k \in \Omega_{i}^{c}(N+1, N)$, and $0 \leq i \leq 2^{N}-1$. Essentially, this means that the equality in (31) is satisfied if and only if for each relay $k$ that decodes erroneously, the $m$ preceding relays also must have decoded erroneously. One can think of this condition as a chain rule, and this leads to the conclusion that the equality holds if and only if for each relay $k$ that decodes in error all the previous relays must have decoded in error. As a result, the only network states that will contribute in the SER expression with terms of order $N+1$ in the SNR are those of the form $\mathbf{S}_{N}=\mathbf{B}_{2^{n}-1, N}, 0 \leq n \leq N$. For example, a network state of the form $\mathbf{S}_{N}=[0, \ldots, 0,1, \ldots, 1]$ will contribute to the term in the SER with SNR raised to the order $N+1$, and a network state $\mathbf{S}_{N}=[0, \ldots, 0,1,1,0,1, \ldots, 1]$ will contribute to the term in the SER with SNR raised to an exponent larger than $(N+1)$ depending on $m$. Therefore, only $N+1$ states of the network have SER terms that decay as $1 / \mathrm{SNR}^{N+1}$ and the rest of the network states decay with faster rates, hence these $N+1$ terms will dominate over the SER expression at high enough SNR.

In order to write the approximate expression for the SER corresponding to these $N+1$ terms, we need to note the following points that can be deduced from the previous analysis. As described previously, in order for the equality in (31) to hold, the following set of conditions must be satisfied. First, since for any relay that decodes erroneously all the previous $m$ relays must have decoded in error, we have

$$
\Omega_{i}(k, m)=\Phi
$$

for all $k \in \Omega_{i}^{c}(N+1, N)$, where $\Phi$ is the empty set. Second, for these $N+1$ states that satisfy the equality in (31), the set $\Omega_{i}^{c}(N+1, N)$ takes one of the following forms:

$$
\Omega_{i}^{c}(N+1, N) \in\{\Phi,\{1\},\{1,2\}, \ldots,\{1,2, \ldots, N\}\} .
$$

For example, $\Omega_{i}^{c}(N+1, N)=\{1,2, \ldots, k\}$ denotes the state in which only the first $k$ relays decoded erroneously. Accordingly, its cardinality, denoted by $\left|\Omega_{i}^{c}(N+1, N)\right|$, takes one of the following values:

$$
\left|\Omega_{i}^{c}(N+1, N)\right| \in\{0,1,2, \ldots, N\}
$$

$$
P_{\mathrm{SER}}(m) \simeq \sum_{i=0}^{2^{N}-1} \frac{\left(\frac{N_{o}}{P}\right)^{d_{i}} g_{q}\left(1+\left|\Omega_{i}(N+1, N)\right|\right) \prod_{k \in \Omega_{i}^{c}(N+1, N)} g_{q}\left(1+\left|\Omega_{i}(k, m)\right|\right)}{b_{q}^{d_{i}} a_{0}^{1+\left|\Omega_{i}^{c}(N+1, N)\right|} \sigma_{s, d}^{2} \prod_{j \in \Omega_{i}(N+1, N)} a_{j} \sigma_{r_{j}, d}^{2} \prod_{k \in \Omega_{i}^{c}(N+1, N)} \sigma_{s, r_{k}}^{2} \prod_{l \in \Omega_{i}(k, m)} a_{l} \sigma_{r_{l}, r_{k}}^{2}}
$$


Thus, only the $N+1$ states determined from the previous conditions will contribute to the SER expression at high SNR because they decay as $1 / \mathrm{SNR}^{N+1}$, which is the slowest decaying rate as seen from (31). From (30) and (32)-(34), the conditional SER for any of these states, e.g., $\Omega_{i}^{c}(N+1, N)=\{1,2, \ldots, k\}$, can be determined as follows:

$$
\operatorname{SER}_{k}(m)=\frac{\left(\frac{\mathcal{N}_{o}}{P}\right)^{N+1} g_{q}(N-k+1) g_{q}^{k}(1)}{b_{q}^{N+1} \sigma_{s, d}^{2} a_{o}^{1+k} \prod_{j \in \Omega_{i}(N+1, N)} a_{j} \sigma_{r_{j}, d}^{2} \prod_{l=1}^{k} \sigma_{s, r_{l}}^{2}} .
$$

Summing (35) over the $N+1$ states in (33), we can further determine the approximate expression for the SER in Theorem 2.

Theorem 2: At high enough SNR, the SER of an $N$-relay decode-and-forward cooperative diversity network employing cooperation scheme $\mathcal{C}(m)$ and utilizing MPSK or MQAM modulation can be approximated by

$$
P_{\mathrm{SER}}(m) \simeq \frac{\left(\frac{\mathcal{N}_{o}}{P}\right)^{N+1}}{b_{q}^{N+1} \sigma_{s, d}^{2}} \sum_{j=1}^{N+1} \frac{g_{q}(N-j+2) g_{q}^{j-1}(1)}{a_{0}^{j} \prod_{i=j}^{N} a_{i} \sigma_{r_{i}, d}^{2} \prod_{l=1}^{j-1} \sigma_{s, r_{l}}^{2}} .
$$

A very important point to be noticed from Theorem 2 is that the approximate SER expression in (36) does not depend on $m$, the class parameter. Hence, the whole class of cooperative diversity protocols $\{\mathcal{C}(m)\}_{m=1}^{N-1}$ shares the same asymptotic performance at high enough SNR. The results obtained in Theorem 2 illustrate that utilizing the simplest scheme, namely, scenario $\mathcal{C}(1)$, results in the same asymptotic SER performance as the most sophisticated scheme, namely, $\mathcal{C}(N-1)$. This motivates us to utilize scenario $\mathcal{C}(1)$ as a cooperative protocol for multinode wireless networks employing decode-and-forward relaying. The simplicity behind scenario $\mathcal{C}(1)$ is due to the fact that it does not require each relay to estimate the CSI for all the previous relays as in scenario $\mathcal{C}(N-1)$. It only requires each relay to know the CSI to the previous relay and the destination thus saving a lot in the channel-estimation computations.

In the following, we determine roughly the savings in the computations needed for channel estimation when using scenario $\mathcal{C}(1)$ as opposed to scenario $\mathcal{C}(N-1)$ by computing the number of channels needed to be estimated in each case. The number of channels needed to be estimated in scenario $\mathcal{C}(1)$ is given by

$$
n_{h, 1}=3 N
$$

where $N$ is the number of relays forwarding for the source. This value accounts for the $N+1$ channels estimated at the destination and $2 N-1$ channels estimated by the $N$ relays; the first relay estimates only one channel. In scenario $\mathcal{C}(N-1)$, the $k$ th relay estimates $k$ channels, and thus the amount of computations for this case is given by

$$
n_{h, N-1}=\frac{1}{2}\left[N^{2}+3 N+1\right]
$$

From (37) and (38), the savings in the computations needed for channel estimation when using scenario $\mathcal{C}(1)$ as opposed to scenario $\mathcal{C}(N-1)$ are given by

$$
\frac{n_{h, 1}}{n_{h, N-1}}=\frac{6 N}{N^{2}+3 N+1} \text {. }
$$

The previous ratio approaches 0 in the limit as $N$ tends to $\infty$. Hence, utilizing scenario $\mathcal{C}(1)$ will reduce the protocol complexity while having the same asymptotic performance as the best possible scenario.

\section{B. Diversity Order and Cooperation Gain}

The philosophy before employing cooperative diversity techniques in wireless networks is to form virtual MIMO systems from separated single-antenna terminals. The aim behind this is to emulate the performance gains that can be achieved in point-to-point communications when employing MIMO systems. Two well-known factors that describe the performance of the system are the diversity order and coding gain of the transmit diversity scheme. To define these terms, the SER can be written in the following form:

$$
P_{\mathrm{SER}} \sim(\Delta \cdot \mathrm{SNR})^{-d}
$$

The constant $\Delta$ which multiplies the SNR denotes the coding gain of the scheme, and the exponent $d$ denotes the diversity order of the system.

In the cooperative diversity schemes considered in this paper, the relays simply repeat the decoded information, and, thus, we do not really have the notion of coding; although it can still be seen as a repetition coding scheme. Hence, we will donate the constant $\Delta$ that multiplies the SNR by the cooperation gain. From (36) in Theorem 2, the following observations can be deduced from the previous relation.

- It is clear that the diversity order of the system is given by $d=N+1$, which indicates that the cooperative diversity schemes described in Section II achieves full diversity order in the number of cooperating terminals, the source and the $N$ relays.

- The cooperation gain of the system is given by

$\Delta=\left[\frac{1}{b_{q}^{N+1} \sigma_{s, d}^{2}} \sum_{j=1}^{N+1} \frac{g_{q}(N-j+2) g_{q}^{j-1}(1)}{a_{0}^{j} \prod_{i=j}^{N} a_{i} \sigma_{r_{i}, d}^{2} \prod_{l=1}^{j-1} \sigma_{s, r_{l}}^{2}}\right]^{-1 /(N+1)}$.

In order to validate the accuracy of the derived approximate SER we conducted some simulation experiments. Throughout all the simulations, and without loss of generality, the channel gains are assumed to be unity and the noise variance is taken to be $N_{o}=1$. Fig. 4 considers scenario $\mathcal{C}(1)$ and depicts the SER performance versus $P / N_{o}$ for QPSK signalling. The transmitting power $P$ is fixed for different number of cooperating relays in the network. The results reveal that the derived approximations for the SER are tight at high enough SNR. Regarding scenario $\mathcal{C}(N-1)$, we considered the $N=3$ relays case. Fig. 5 depicts the SER performance for QPSK and 16 QAM modulation. The results for scenario $\mathcal{C}(1)$ under the same simulation setup are included for comparison. It can be seen from the results that there is a very small gap between the SER performance of scenarios $\mathcal{C}(1)$ and $\mathcal{C}(N-1)$, and that they almost merge together at high enough SNR. This confirms our observations that utilizing scenario $\mathcal{C}(1)$ can deliver the required SER performance for a fairly wide range of SNR, hence, saving a lot in terms of channel estimation, thus computational complexity, requirements to implement the protocol. 


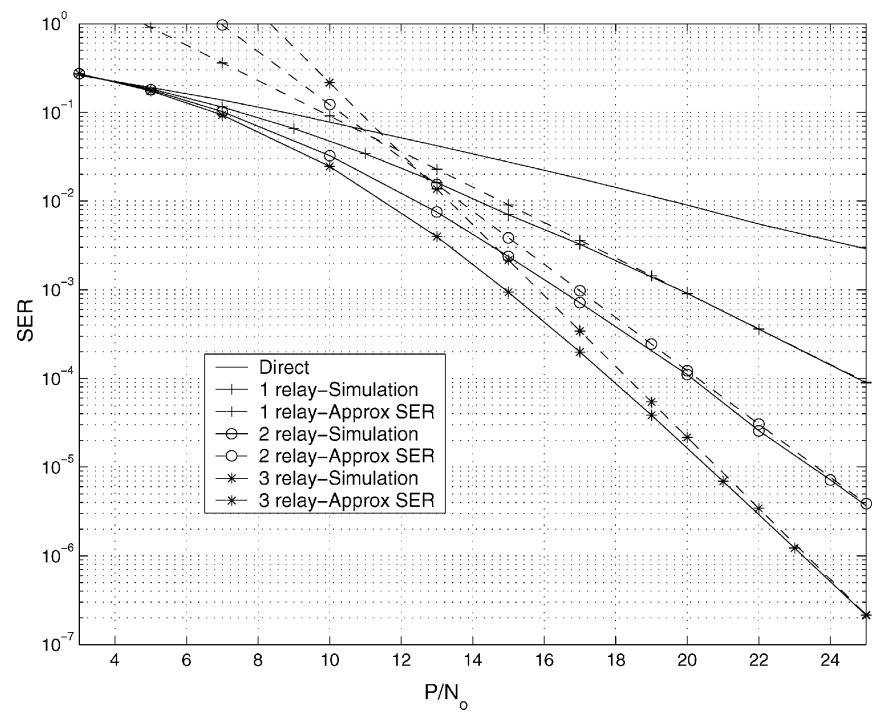

Fig. 4. Comparison between the approximate SER in (36), and the simulated SER for different number of relays. The cooperation protocol utilized is $\mathcal{C}(1)$ and the modulation scheme is QPSK.

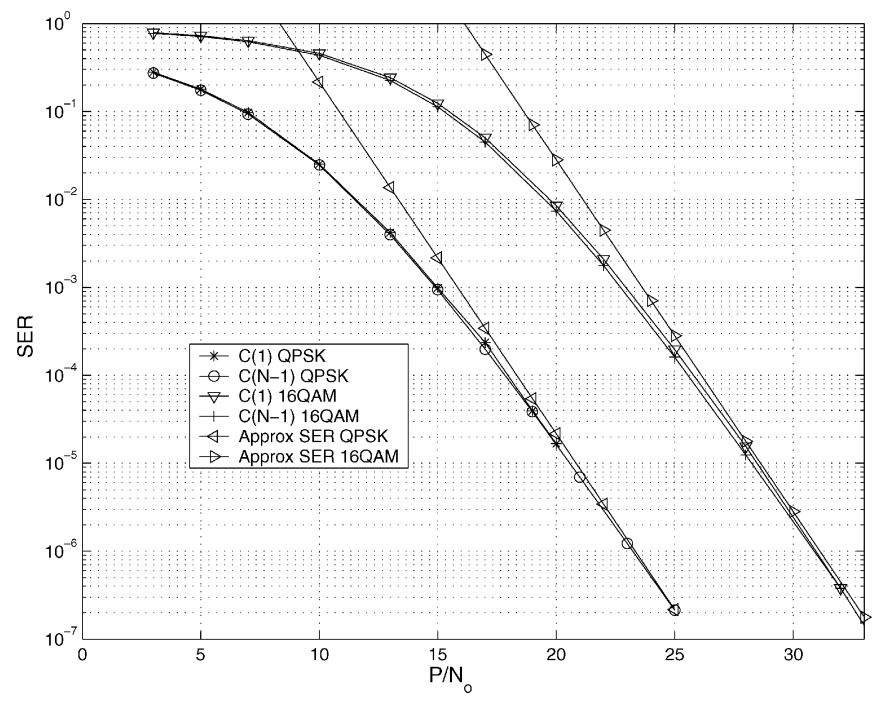

Fig. 5. Comparison between the performance of schemes $\mathcal{C}(1)$ and $\mathcal{C}(N-1)$ for both QPSK and 16 QAM modulation, $N=3$.

\section{Bandwidth Efficiency Versus Diversity Gain}

Up to this point, we did not take into account the bandwidth (BW) efficiency as another important factor to determine the performance besides the SER. Increasing the number of relays reduces the BW efficiency of the system, as the source uses only a fraction of the total available degrees of freedom to transmit the information. There is a tradeoff between the diversity gain and the BW efficiency of the system, as higher diversity gain is usually translated into utilizing the available degrees of freedom to transmit more copies of the same message which reduces the BW efficiency of the system. In order to have a fair comparison, we will fix the BW efficiency throughout the simulations. In order to achieve this, larger signal constellations are utilized with larger number of cooperating relays. For the direct transmission case, BPSK is used as a benchmark to achieve bandwidth efficiency of $1 \mathrm{bit} / \mathrm{channel}$ use. QPSK is used with the

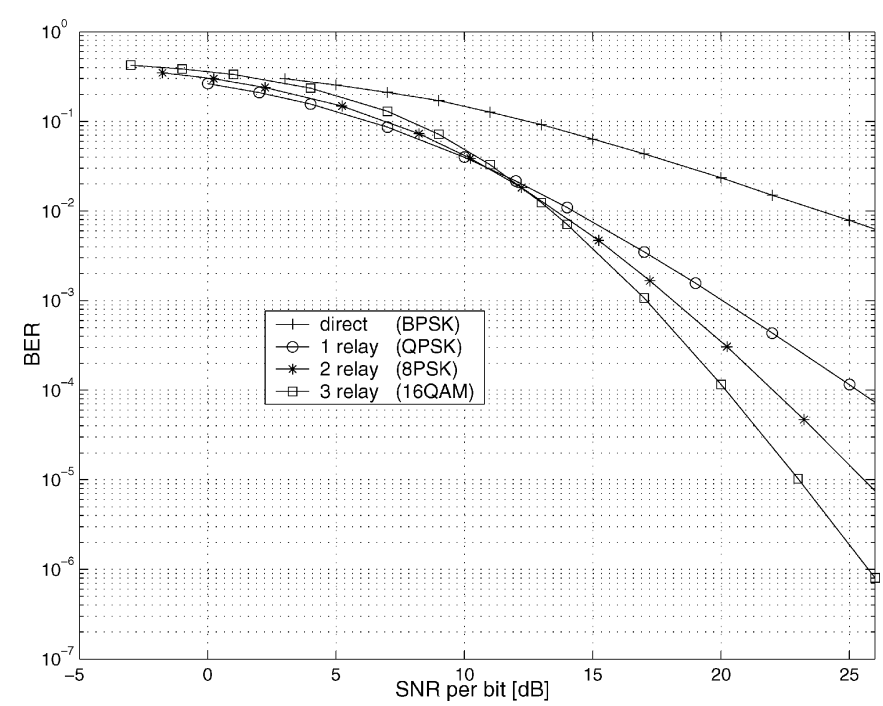

Fig. 6. BER performance comparison between different numbers of cooperating relays taking into account the BW efficiency $\mathcal{C}(1)$.

$N=1$ relay case, 8 PSK with $N=2$ relays, and 16 QAM with $N=3$ relays. In all of the aforementioned cases, the achieved BW efficiency is 1 bit/channel use. Fig. 6 depicts the bit error rate (BER) versus SNR per bit in $\mathrm{dB}$ for $N=1,2,3$ relays along with the direct transmission case. The results reveal that at low SNR, lower number of nodes achieves better performance due to the BW efficiency loss incurred with utilizing larger number of cooperating nodes.

Another important point of concern is how the performance of cooperative diversity compare to that of time diversity without relaying under the same bandwidth efficiency. For example, if the target diversity gain is $N+1$, then cooperation requires the employment of $N$ relays, while in time diversity, the source simply repeats the information for $N+1$ successive time slots. Two factors can lead to cooperation yielding better performance than time diversity. The first is that the cooperation gain of cooperative diversity (41) can be considerably higher than that of time diversity if the propagation path loss is taken into account. This is because the relay nodes are usually closer to the destination node than the source itself, which results in less propagation path loss in the relays-destination links compared to the source-destination link. This is a natural gain offered by cooperation because of the distributed natural of the formed virtual array, and this is the same reason multihop communications offer more energy efficient transmission in general. The second factor which can lead to cooperation being a more attractive scheme than time diversity is that the spatial links between different nodes in the network fade independently, again because of the distributed nature of the formed virtual array, which leads to full diversity gain. In time diversity, however, full diversity gain is not guaranteed as there might be time correlation between successive time slots. This correlation is well modeled by a first-order Markov chain [18]. To illustrate the previously described factors better, we compare the SER performance of time and cooperative diversity in Fig. 7. The desired diversity gain is 3 . The time correlation factor for the first-order Markov model is taken equal to $\rho=0.9,0.7,0.3,0.1$. The two relays are 


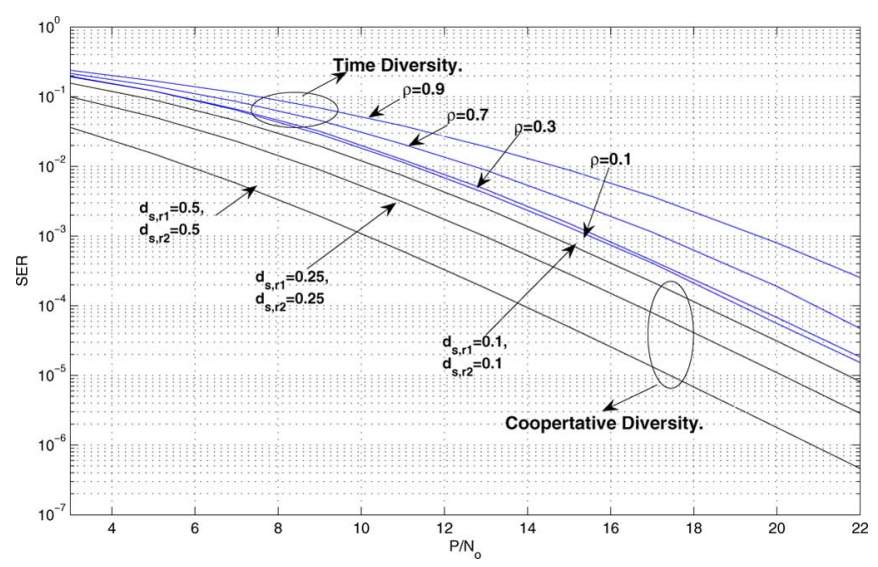

Fig. 7. Comparison between the SER performance of time diversity without any relaying and cooperative diversity. Two relays are utilized for cooperation and correspondingly three time slots for time diversity. The first-order Markov model is utilized to account for time correlation, and different relays' positions are depicted with the source-destination distance normalized.

taken in different positions as illustrated in the figure to illustrate different coding gains. It is clear from Fig. 7 that cooperative diversity can offer better performance than time diversity because of the higher possible coding gain that depends on the relay positions, and the degradation in the achieved performance of time diversity due to the correlation factor $\rho$.

\section{Optimal POWER Allocation}

In this section, we try to find the optimal power-allocation strategy for the multinode cooperative scenarios considered in the previous sections. The approximate SER formula derived in (36) is a function of the power allocated at the source and the $N$ relays. For a fixed transmission power budget $P$, the power should be allocated optimally at the different nodes in order to minimize the SER.

Since the approximation in (36) is tight at high enough SNR, we use it to determine the asymptotic optimum power allocation; also, we drop the parameter $m$ as the asymptotic SER performance is independent of it. The SER can be written in terms of the power ratios allocated at the transmitting nodes as follows:

$$
\begin{aligned}
P_{\mathrm{SER}} \simeq\left(\frac{P}{N_{o}}\right)^{-(N+1)} & \frac{1}{b_{q}^{N+1} \sigma_{s, d}^{2}} \\
& \times \sum_{j=1}^{N+1} \frac{g_{q}(N-j+2) g_{q}^{j-1}(1)}{a_{0}^{j} \prod_{i=j}^{N} a_{i} \sigma_{r_{i}, d}^{2} \prod_{k=1}^{j-1} \sigma_{s, r_{k}}^{2}}
\end{aligned}
$$

The nonlinear optimization problem can be formulated as follows:

$$
\mathbf{a}_{\mathrm{opt}}=\arg \min _{\mathbf{a}} P_{\mathrm{SER}}
$$

$$
\text { subject to } a_{i} \geq 0(0 \leq i \leq N), \quad \sum_{i=0}^{N} a_{i}=1
$$

where $\mathbf{a}=\left[a_{0}, a_{1}, \ldots, a_{N}\right]$ is the power allocating vector. The Lagrangian of this problem can be written as

$$
L=P_{\mathrm{SER}}+\nu\left(\sum_{i=0}^{N} a_{i}-1\right)-\sum_{j=0}^{N} \beta_{j} a_{j}
$$

where the $\beta$ 's act as slack variables.

Although this nonlinear optimization problem should be solved numerically, in general, there are some insights which can be drawn out of it. Applying first-order optimality conditions, we can show that the optimum power-allocation vector $\mathbf{a}_{\text {opt }}$ must satisfy the following necessary conditions:

$$
\frac{\partial P_{\mathrm{SER}}}{\partial a_{i}}=\frac{\partial P_{\mathrm{SER}}}{\partial a_{j}}, \quad i, j \in\{0,1,2, \ldots, N\} .
$$

Next, we solve these equations simultaneously to get the relations between the optimal power allocations at different nodes. To simplify the notations, let $\mu_{j}$ denote the constant quantity inside the summation in (42), i.e.,

$$
\mu_{j}=\left(\frac{P}{N_{o}}\right)^{-(N+1)} \frac{g_{q}(N-j+2) g_{q}^{j-1}(1)}{b_{q}^{N+1} \sigma_{s, d}^{2} \prod_{i=j}^{N} \sigma_{r_{i}, d}^{2} \prod_{k=1}^{j-1} \sigma_{s, r_{k}}^{2}} .
$$

The derivative of the SER with respect to $a_{0}$ is given by

$$
\frac{\partial P_{\mathrm{SER}}}{\partial a_{0}}=\sum_{j=1}^{N+1} \frac{-j \mu_{j}}{a_{0}^{j+1} \prod_{i=j}^{N} a_{i}}
$$

while the derivative with respect to $a_{k}, 1 \leq k \leq N$ is given by

$$
\frac{\partial P_{\mathrm{SER}}}{\partial a_{k}}=\sum_{j=1}^{k} \frac{-\mu_{j}}{a_{0}^{j} a_{k} \prod_{i=j}^{N} a_{i}}
$$

where the summation is to the $k$ th term only as $a_{k}$ does not appear in the terms from $k+1$ to $N$. Using (45), we equate the derivatives of the SER with respect to any two consecutive variables $a_{k}$ and $a_{k+1}, 1 \leq k \leq N-1$, as follows:

$$
\sum_{j=1}^{k} \frac{-\mu_{j}}{a_{0}^{j} a_{k} \prod_{i=j}^{N} a_{i}}=\sum_{j=1}^{k+1} \frac{-\mu_{j}}{a_{0}^{j} a_{k+1} \prod_{i=j}^{N} a_{i}}
$$

Rearranging the terms in (49), we get

$$
\frac{a_{k+1}-a_{k}}{a_{k} a_{k+1}} \sum_{j=1}^{k} \frac{\mu_{j}}{a_{0}^{j} \prod_{i=j}^{N} a_{i}}=\frac{\mu_{k+1}}{a_{0}^{j} a_{k+1} \prod_{i=k+1}^{N} a_{i}} .
$$

Since both sides of (50) are positive, we conclude that $a_{k+1} \geq$ $a_{k}$ for any $k=1,2, \ldots, N$. Similarly, we can show that $a_{o} \geq$ $a_{k}$ for all $1 \leq k \leq N$. Hence, solving the optimality conditions simultaneously, we get the following relationships between the powers allocated at different nodes:

$$
P_{0} \geq P_{N} \geq P_{N-1} \geq \cdots \geq P_{1}
$$

The previous set of inequalities demonstrates an important concept: Power is allocated at different nodes according to the 


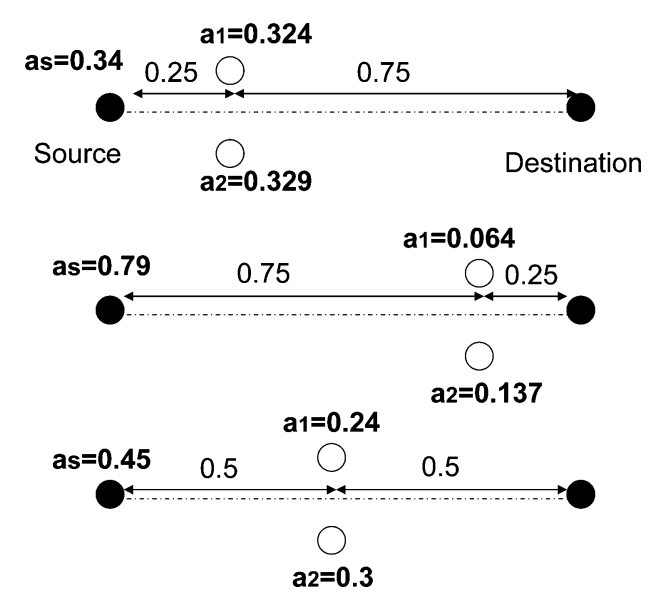

Fig. 8. Optimal power allocation for $N=2$ relays under different relays positions.

received signal quality at these node. We refer to the quality of the signal copy at a node as the reliability of the node, thus the more reliable the node the more power allocated to this node. To further illustrate this concept, the $N+1$ cooperative nodes form a virtual $(N+1) \times 1$ MIMO system. The difference between this virtual array and a conventional point-to-point MIMO system is that in conventional point-to-point communications all the antenna elements at the transmitter are allocated at the same place and, hence, all the antenna elements can acquire the original signal. In a virtual array, the antenna elements constituting the array (the cooperating nodes) are not allocated at the same place and the channels among them are noisy. The source is the most reliable node as it has the original copy of the signal and, thus, it should be allocated at the highest share of the power. According to the cooperation protocol described in Section II, each relay combines the signal received from the source and the previous relays. As a result, each relay is more reliable than the previous relay, and, hence, the $N$ th relay is the most reliable node and is allocated the largest ratio of the power after the source, and the first relay is the least reliable and is allocated the smallest ratio of the transmitted power. Another important point to notice is that the channel quality of the direct link between the source and the destination $\sigma_{s, d}^{2}$ is a common factor in the $\mu_{j}$ 's that appear in (50); hence, the optimal power allocation does not depend on it.

To illustrate the effect of relay position on the values of the optimal power-allocation ratios at the source and relay nodes, we consider a two-relays scenario in Fig. 8. The two relays are taken in three different positions, close to the source, close to the destination, and in the middle between the source and the destination. In the first scenario, almost equal power allocation between the three nodes is optimal. When the relays are closer to the destination, more power is allocated to the source node, but still the second relay has a higher portion of the power relative to the first one. Similarly, in the last scenario the last relay has more power than the first one. These results reveal the fact that the further the relays from the source node are the less power is allocated to the relays as they become less reliable, while as the relays become closer to the source, equal power allocation

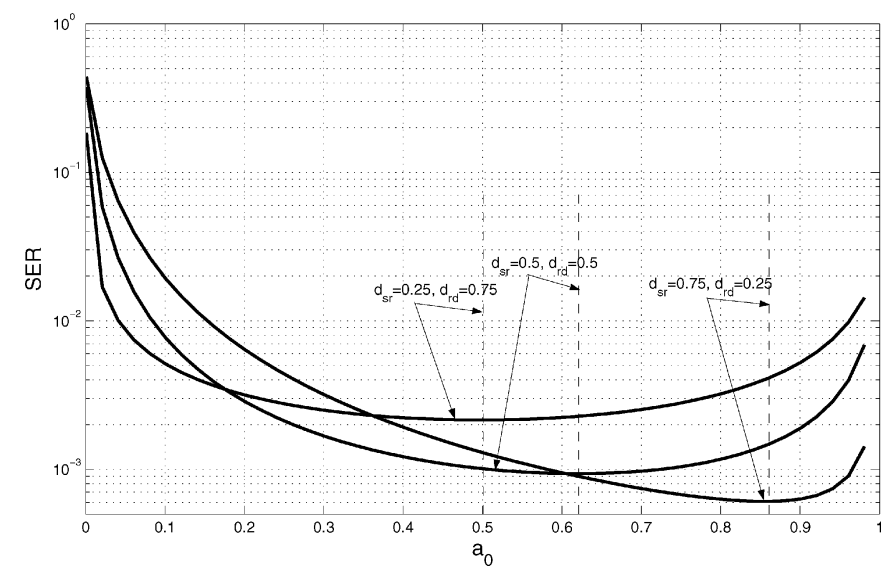

Fig. 9. SER versus power-allocation ratio at the source node for different relay positions.

becomes near optimal. This is similar to the results of optimal power allocation for distributed space-time coding in [15].

There are a few special cases of practical interest that permit a closed-form solution for the optimization problem in (43), and they are discussed in the sequel.

\section{A. Single-Relay Scenario}

For the $N=1$ relay scenario [7], the optimization problem in (43) admits closed-form expression. The SER for this case is simply given as

$$
P_{\mathrm{SER}}=\left(\frac{P}{N_{o}}\right)^{-2} \frac{1}{b_{q}^{2} \sigma_{s, d}^{2}}\left(\frac{g_{q}(2)}{a_{0} a_{1} \sigma_{r_{1}, d}^{2}}+\frac{g_{q}^{2}(1)}{a_{0}^{2} \sigma_{s, r_{1}}^{2}}\right) .
$$

Solving the optimization problem for this case leads to the following solution for the optimal power allocation:

$$
\begin{aligned}
& a_{0}=\frac{\sigma_{s, r_{1}}+\sqrt{\sigma_{s, r_{1}}^{2}+8 \frac{g_{q}^{2}(1)}{g_{q}(2)} \sigma_{r_{1}, d}^{2}}}{3 \sigma_{s, r_{1}}+\sqrt{\sigma_{s, r_{1}}^{2}+8 \frac{g_{q}^{2}(1)}{g_{q}(2)} \sigma_{r_{1}, d}^{2}}} \\
& a_{1}=\frac{2 \sigma_{s, r_{1}}}{3 \sigma_{s, r_{1}}+\sqrt{\sigma_{s, r_{1}}^{2}+8 \frac{g_{q}^{2}(1)}{g_{q}(2)} \sigma_{r_{1}, d}^{2}}} .
\end{aligned}
$$

To study the effect of relay position on the optimal power allocation, we depict in Fig. 9 the SER performance of a single-relay scenario versus the power allocation at the source node $a_{0}$ for different relay positions. The first observation that the figure reveals is that the SER performance is relatively flat around equal power allocation when the relay is not very close to the destination - an observation that was also made for distributed space-time coding in [15]. Another observation to notice here is that as the relay becomes closer to the destination, the value of the optimal power allocation at the source node $a_{0}$ approaches 1 , which means that as the relay node becomes less reliable more power should be allocated to the source node.

\section{B. Networks With Linear Topologies}

The propagation path loss will be taken into account here. The channel attenuation between any two nodes $\sigma_{i, j}^{2}$ depends 
on the distance between these two nodes $d_{i, j}$ as follows: $\sigma_{i, j}^{2} \propto$ $d^{-\alpha}$, where $\alpha$ is the propagation constant. For a linear network topology, the most significant channel gains are for the channels between the source and the first relay $\sigma_{s, r_{1}}^{2}$, and that between the last relay and the destination $\sigma_{r_{N}, d}^{2}$; the other channel gains are considerably smaller than these two channels. In the SER expression in (36), these two terms appear as a product in all the terms except the first and the last terms. Hence, these two terms dominate the SER expression, and we can further approximate the SER in this case as follows:

$$
P_{\mathrm{SER}} \simeq \frac{\left(\frac{\mathcal{N}_{o}}{P}\right)^{N+1}}{b_{q}^{N+1} \sigma_{s, d}^{2}}\left[\frac{g_{q}(N+1)}{a_{0} \prod_{i=1}^{N} a_{i} \sigma_{r_{i}, d}^{2}}+\frac{g_{q}^{N+1}(1)}{a_{0}^{N+1} \prod_{i=1}^{N} \sigma_{s, r_{i}}^{2}}\right]
$$

Taking the power constraint into consideration, the Lagrangian of (54) can be written as

$$
L(\mathbf{a})=\frac{\mu_{1}}{a_{o} \prod_{i=1}^{N} a_{i}}+\frac{\mu_{N+1}}{a_{o}^{N+1}}+\lambda\left(\sum_{i=0}^{N} a_{i}-1\right)
$$

where the constants $\mu_{1}$ and $\mu_{N+1}$ are defined in (46).

Taking the partial derivatives of the Lagrangian with respect to $a_{j}, 1 \leq j \leq N$, and equating with 0 , we get

$$
a_{j}=\frac{\mu_{1}}{\lambda a_{o} \prod_{i=1}^{N} a_{i}} .
$$

Thus, we deduce that the power allocated to all of the relays are equal. Let the constant $\kappa$ be defined as follows:

$$
\kappa=\frac{P_{0}-P_{j}}{P_{j}} .
$$

From (57), along with the power constraint, we get

$$
P_{j}=\frac{1}{1+\kappa+N} P \quad P_{0}=\frac{1+\kappa}{1+\kappa+N} P .
$$

To find the optimum value for $\kappa$, substitute (58) into the expression for the SER in (54) to get

$P_{\mathrm{SER}} \simeq \frac{\mu_{1}(1+\kappa)^{N}(1+\kappa+N)^{N+1}+\mu_{N+1}(1+\kappa+N)^{N+1}}{(1+\kappa)^{N+1}}$.

Differentiating (59) and equating to 0 , we can find that the optimum $\kappa$ satisfies the equation $\kappa(1+\kappa)^{N}=A$, in which $A$ is a constant given by $(N+1)\left(g_{q}^{N+1}(1) \prod_{i=1}^{N} \sigma_{r_{i}, d}^{2} / g_{q}(N+1) \prod_{i=1}^{N} \sigma_{s, r_{i}}^{2}\right)$.

From the previous analysis, the optimal power allocation for a linear network can be found in Theorem 3 .

Theorem 3: The optimal power allocation for a linear network that minimizes the SER expression in (54) is as follows:

$$
P_{0}=\frac{1+\kappa}{1+\kappa+N} P \quad P_{i}=\frac{1}{1+\kappa+N} P, \quad 1 \leq i \leq N
$$

where $\kappa$ is found through solving the equation $\kappa(1+$ $\kappa)^{N}=A$, in which $A$ is a constant given by $(N+$ 1) $\left(g_{q}^{N+1}(1) \prod_{i=1}^{N} \sigma_{r_{i}, d}^{2} /\left(g_{q}(N+1) \prod_{i=1}^{N} \sigma_{s, r_{i}}^{2}\right)\right)$.

Theorem 3 agrees with optimality conditions we found for the general problem in (51). Also, it shows an interesting property
TABLE I

COMPARISON BETWEen Optimal POWER Allocation Via EXHaustive SEARCH AND ANALYTICAL RESUlTS. $N=3$ RELAYS, UNIFORM NETWORK TOPOLOGY

\begin{tabular}{|c|c|}
\hline Exhaustive Search & Analytical Results \\
\hline \hline$P_{0}=0.31 P$ & $P_{0}=0.31 P$ \\
\hline$P_{1}=0.23 P$ & $P_{1}=0.23 P$ \\
\hline$P_{2}=0.23 P$ & $P_{2}=0.23 P$ \\
\hline$P_{3}=0.23 P$ & $P_{3}=0.23 P$ \\
\hline
\end{tabular}

that in linear network topologies equal power allocation at the relays is asymptotically optimal.

\section{Relays Located Near the Source or the Destination}

The cooperating relays can be chosen to be closer to the source than to the destination, in order for the $N+1$ cooperating nodes to mimic a multi-input-single-output (MISO) transmit antenna diversity system. This case is of special interest as it was shown in [14] that decode-and-forward relaying can be a capacity achieving scheme when the relays are taken to be closer to the source and it has the best performance compared to amplify-and-forward and compress-and-forward relaying in this case. In order to model this scenario in our SER formulation, we will consider the channel gains from the source to the relays that have higher gains than those from the relays to the destination, i.e., $\sigma_{s, r_{i}}^{2} \gg \sigma_{r_{i}, d}^{2}$ for $1 \leq i \leq N$. Taking this into account, the approximate SER expression in (36) can be further approximated as

$$
P_{\mathrm{SER}} \simeq \frac{N_{o}^{N+1} g_{q}(N+1)}{b_{q}^{N+1} \sigma_{s, d}^{2} P^{N+1} a_{0} \prod_{i=1}^{N} a_{i} \sigma_{r_{i}, d}^{2}} .
$$

It is clear from (61) that the SER depends equally on the power allocated to all nodes including the source, and thus the optimal power-allocation strategy for this case is simply given by

$$
P_{0}=P_{i}=\frac{P}{N+1}, \quad 1 \leq i \leq N .
$$

This result is intuitively meaningful as all the relays are located near to the source and thus they all have high reliability and are allocated equal power as if they form a conventional antenna array.

Now we consider the opposite scenario in which all the relays are located near the destination. In this case, the channels between the relays and the destination are of a higher quality and higher gain, than those between the source and the relays, i.e., $\sigma_{r_{i}, d}^{2} \gg \sigma_{s, r_{i}}^{2}$ for $1 \leq i \leq N$. In this case, the SER can be approximated as

$$
P_{\mathrm{SER}} \simeq \frac{N_{o}^{N+1} g(1)^{N+1}}{b_{q}^{N+1} \sigma_{s, d}^{2} P_{0}^{N+1} \prod_{k=1}^{N} \sigma_{s, r_{k}}^{2}}
$$

The SER in (63) is not a function of the power allocated at the cooperating relays, and thus the optimal power allocation in this case is simply $P_{0}=P$, i.e., allocating all the available power at the source. This result is very interesting as it reveals a very important concepts: If the relays are located closer to the destination than to the transmitter then direct transmission can lead 
TABLE II

Comparison Between Optimal Power Allocation Via Exhaustive Search and Analytical Results. $N=3$ Relays: (A) All Relays Near the Source and (B) All Relays Near The Destination

\begin{tabular}{|c|c||c|c|}
\hline (A) Exhaustive Search & Analy. Results & (B) Exhaustive Search & Analy. Results \\
\hline \hline$P_{0}=0.25 P$ & $P_{0}=0.25 P$ & $P_{0}=0.875 P$ & $P_{0}=P$ \\
\hline$P_{1}=0.25 P$ & $P_{1}=0.25 P$ & $P_{1}=0.015 P$ & $P_{1}=0$ \\
\hline$P_{2}=0.25 P$ & $P_{2}=0.25 P$ & $P_{2}=0.035 P$ & $P_{2}=0$ \\
\hline$P_{3}=0.25 P$ & $P_{3}=0.25 P$ & $P_{3}=0.075 P$ & $P_{3}=0$ \\
\hline
\end{tabular}

better performance than decode-and-forward relaying. This is also consistent with the results in [14] in which it was shown that the performance of the decode-and-forward strategy degrades significantly when the relays get closer to the destination. This result can be intuitively interpreted as follows: The farther the relays from the source are, the more noisy the channels between them become, and the less reliable the signals received by those relays are to the extent that we cannot rely on them on forwarding copies of the signal to the destination.

\section{Numerical Examples}

In this section, we present some numerical results to verify the analytical results for the optimal power-allocation problem for the considered network topologies. The effect of the geometry on the channel links qualities is taken into consideration. We assume that the channel variance between any two nodes is proportional to the distance between them, more specifically $\sigma_{i, j}^{2} \propto d_{i, j}^{-\alpha}$, where $\alpha$ is determined by the propagation environment is taken equal to 4 throughout our simulations. We provide comparisons between the optimal power allocation via exhaustive search to minimize the SER expression in (36), and optimal power allocation provided by the closed-form expressions provided in this section.

First, for the linear network topology, we consider a uniform linear network, i.e., $d_{s, r_{1}}=d_{r_{1}, r_{2}}=\cdots=d_{r_{N}, d}$. The variance of the direct link between the source and the destination is taken to be $\sigma_{s, d}^{2}=1$. Table I demonstrates the results for $N=3$ relays. Second, for the case when all the relays are near the source, the channel links are taken to be $\sigma_{s, r_{i}}^{2}=\sigma_{r_{i}, r_{j}}^{2}=10$, while $\sigma_{s, d}^{2}=\sigma_{r_{i}, d}^{2}=0.1$. Finally, for the case when all of the relays are near the destination, the channel link qualities are taken to be $\sigma_{s, d}^{2}=\sigma_{s, r_{i}}^{2}=0.1$, while $\sigma_{r_{i}, r_{j}}^{2}=\sigma_{r_{i}, d}^{2}=10$. Table II illustrates the results for $N=3$ relays for the two previous cases. In all of the provided numerical examples, it is clear that the optimal power allocations obtained via exhaustive search agree with that via analytical results for all the considered scenarios. Also, the numerical results show that the optimal power allocation obtained via exhaustive search has the same ordering as the one we got in (51).

\section{CONCLUSION}

In this paper, we propose a class of cooperative diversity protocols for multinode wireless networks employing decode-and-forward relaying. This class of protocols consists of schemes in which each relay can combine the signals arriving from an arbitrary but fixed number of previous relays along with that received from the source. We derive exact expressions for the SER of a general cooperation scheme for both MPSK and MQAM modulation. Also, we provide approximations for the SER which are shown to be tight at high enough SNR. Our theoretical analysis reveals a very interesting result: This class of cooperative protocols shares the same asymptotic performance at high enough SNR. Thus, the performance of a simple cooperation scenario in which each relay combines the signals arriving from the previous relay and the source is asymptotically exactly the same as that for the most complicated scenario in which each relay combines the signals arriving from all the previous relays and the source. The analysis also reveals that the proposed protocols achieve full diversity gain in the number of cooperating terminals. Moreover, we formulate the optimal power-allocation problem, and show that the optimum power allocated at the nodes for an arbitrary network follow a certain ordering. We find that the optimal power-allocation scheme does not depend on the quality of the direct link between the source and the destination. Furthermore, we provide closed-form solutions for the optimal power allocation for some network topologies of practical interest, and we show through numerical examples that our theoretical results match with the simulation results.

\section{APPENDIX}

In this appendix, we provide a proof for the tightness of the approximations we use to derive the asymptotic SER expressions at high SNR. For space limitations, we include only the proof for a single-relay scenario using MPSK modulations. The proof for the general scenario follows easily in the same footsteps. The purpose for this proof is just to illustrate what we rigorously mean by ignoring the $1 \mathrm{~s}$ in the $F_{q}(\cdot)$ functions in the SER expressions at high SNR.

For the single-relay case, the SER is given by

$$
\begin{aligned}
P_{\mathrm{SER}}= & F_{q}\left(1+\frac{b_{q} P_{0} \sigma_{s, d}^{2}}{\mathcal{N}_{o} \sin ^{2}(\theta)}\right) F_{q}\left(1+\frac{b_{q} P_{0} \sigma_{s, r_{1}}^{2}}{\mathcal{N}_{o} \sin ^{2}(\theta)}\right) \\
& +F_{q}\left[\left(1+\frac{b_{q} P_{0} \sigma_{s, d}^{2}}{\mathcal{N}_{o} \sin ^{2}(\theta)}\right)\left(1+\frac{b_{q} P_{1} \sigma_{r_{1}, d}^{2}}{\mathcal{N}_{o} \sin ^{2}(\theta)}\right)\right] \\
& \times\left[1-F_{q}\left(1+\frac{b_{q} P_{0} \sigma_{s, r_{1}}^{2}}{\mathcal{N}_{o} \sin ^{2}(\theta)}\right)\right] .
\end{aligned}
$$




$$
\begin{aligned}
& \lim _{x \rightarrow \infty} x^{2} I_{1}(x)=\lim _{x \rightarrow \infty} F_{1}\left(\frac{1}{x}+\frac{b_{1} \sigma_{s, d}^{2}}{\sin ^{2} \theta}\right) F_{1}\left(\frac{1}{x}+\frac{b_{1} \sigma_{s, r_{1}}^{2}}{\sin ^{2} \theta}\right) \\
& =\lim _{x \rightarrow \infty} \frac{1}{\pi^{2}} \int_{0}^{(M-1) \pi / M} \int_{0}^{(M-1) \pi / M} \frac{1}{\left(\frac{1}{x}+\frac{b_{1} \sigma_{s, d}^{2}}{\sin ^{2} \theta_{1}}\right)\left(\frac{1}{x}+\frac{b_{1} \sigma_{s, r_{1}}^{2}}{\sin ^{2} \theta_{2}}\right)} d \theta_{1} d \theta_{2} \\
& =\frac{1}{\pi^{2}} \int_{0}^{(M-1) \pi / M} \int_{0}^{(M-1) \pi / M} \lim _{x \rightarrow \infty} \frac{1}{\left(\frac{1}{x}+\frac{b_{1} \sigma_{s, d}^{2}}{\sin ^{2} \theta_{1}}\right)\left(\frac{1}{x}+\frac{b_{1} \sigma_{s, r_{1}}^{2}}{\sin ^{2} \theta_{2}}\right)} d \theta_{1} d \theta_{2} \\
& =\frac{1}{\pi^{2}} \int_{0}^{(M-1) \pi / M} \int_{0}^{(M-1) \pi / M} \frac{\sin ^{2} \theta_{1} \sin ^{2} \theta_{2}}{b_{1}^{2} \sigma_{s, d}^{2} \sigma_{s, r_{1}}^{2}} d \theta_{1} d \theta_{2}=\frac{g_{1}(1)^{2}}{b_{1}^{2} \sigma_{s, d}^{2} \sigma_{s, r_{1}}^{2}} \\
& \lim _{x, y \rightarrow \infty} x y I_{2}(x, y)=\lim _{x, y \rightarrow \infty} F_{1}\left(\left(\frac{1}{x}+\frac{b_{1} \sigma_{s, d}^{2}}{\sin ^{2} \theta}\right)\left(\frac{1}{y}+\frac{b_{1} \sigma_{r_{1}, d}^{2}}{\sin ^{2} \theta}\right)\right)\left[1-F_{1}\left(1+\frac{x b_{1} \sigma_{s, r_{1}}^{2}}{\sin ^{2} \theta}\right)\right] \\
& =\frac{1}{\pi} \int_{0}^{(M-1) \pi / M} \lim _{x, y \rightarrow \infty} \frac{1}{\left(\frac{1}{x}+\frac{b_{1} \sigma_{s, d}^{2}}{\sin ^{2} \theta}\right)\left(\frac{1}{y}+\frac{b_{1} \sigma_{r_{1}, d}^{2}}{\sin ^{2} \theta}\right)} d \theta \\
& -\frac{1}{\pi^{2}} \int_{0}^{(M-1) \pi / M} \int_{0}^{(M-1) \pi / M} \lim _{x, y \rightarrow \infty} \frac{1}{\left(\frac{1}{x}+\frac{b_{1} \sigma_{s, d}^{2}}{\sin ^{2} \theta_{1}}\right)\left(\frac{1}{y}+\frac{b_{1} \sigma_{r_{1}, d}^{2}}{\sin ^{2} \theta_{1}}\right)\left(1+\frac{x b_{1} \sigma_{s, r_{1}}^{2}}{\sin ^{2} \theta_{2}}\right)} d \theta_{1} d \theta_{2} \\
& =\frac{1}{\pi} \int_{0}^{(M-1) \pi / M} \frac{\sin ^{4} \theta}{b_{1}^{2} \sigma_{s, d}^{2} \sigma_{r_{1}, d}^{2}} d \theta=\frac{g_{1}(2)}{b_{1}^{2} \sigma_{s, d}^{2} \sigma_{r_{1}, d}^{2}}
\end{aligned}
$$

Define the functions $I_{1}(x)$ and $I_{2}(x, y)$ as follows:

$$
\begin{aligned}
I_{1}(x)= & F_{1}\left(1+\frac{b_{1} x \sigma_{s, d}^{2}}{\mathcal{N}_{o} \sin ^{2}(\theta)}\right) F_{1}\left(1+\frac{b_{1} x \sigma_{s, r_{1}}^{2}}{\mathcal{N}_{o} \sin ^{2}(\theta)}\right) \\
I_{2}(x, y)= & F_{1}\left[\left(1+\frac{b_{1} x \sigma_{s, d}^{2}}{\mathcal{N}_{o} \sin ^{2}(\theta)}\right)\left(1+\frac{b_{1} y \sigma_{r_{1}, d}^{2}}{\mathcal{N}_{o} \sin ^{2}(\theta)}\right)\right] \\
& \times\left[1-F_{1}\left(1+\frac{b_{1} x \sigma_{s, r_{1}}^{2}}{\mathcal{N}_{o} \sin ^{2}(\theta)}\right)\right]
\end{aligned}
$$

where the function $F_{q}(\cdot)$ is defined for MPSK $(q=1)$ in (18).

We are now going to prove that

$$
\begin{aligned}
\lim _{x \rightarrow \infty} x^{2} I_{1}(x) & =\frac{g_{1}^{2}(1)}{b_{1}^{2} \sigma_{s, d}^{2} \sigma_{s, r_{1}}^{2}} \\
\lim _{x, y \rightarrow \infty} x y I_{2}(x, y) & =\frac{g_{1}(2)}{b_{1}^{2} \sigma_{s, d}^{2} \sigma_{r_{1}, d}^{2}} .
\end{aligned}
$$

The proofs are shown in the equations at the top of the page, where the function $g_{1}(\cdot)$ is defined in (26). The approximate expression for the SER for the single-relay scenario then follows as provided in the paper (52).

As can be seen, the proof for tightness depends on simple evaluation of some limit functions as the SNR tends to infinity, and the proofs for the multinode case and MQAM follow in the same footsteps.

\section{REFERENCES}

[1] G. J. Foschini and M. Gans, "On the limits of wireless communication in a fading environment when using multiple antennas," Wireless Personal Commun., vol. 6, pp. 311-335, Mar. 1998.

[2] E. Telatar, "Capacity of multi-antenna Gaussian channels," Eur. Trans. Telecom., vol. 10, pp. 585-595, Nov. 1999.

[3] J. N. Laneman, D. N. C. Tse, and G. W. Wornell, "Cooperative diversity in wireless networks: Efficient protocols and outage behavior," IEEE Trans. Inf. Theory, vol. 50, no. 12, pp. 3062-3080, Dec. 2004.

[4] J. N. Laneman and G. W. Wornell, "Distributed space-time coded protocols for exploiting cooperative diversity in wireless networks," IEEE Trans. Inf. Theory, vol. 49, no. 10, pp. 2415-2525, Oct. 2003.

[5] A. Sendonaris, E. Erkip, and B. Aazhang, "User cooperation diversity-Part I: System description," IEEE Trans. Commun., vol. 51, no. 11, pp. 1927-1938, Nov. 2003.

[6] — - "User cooperation diversity—Part II: Implementation aspects and performance analysis," IEEE Trans. Commun., vol. 51, no. 11, pp. 1939-1948, Nov. 2003.

[7] W. Su, A. K. Sadek, and K. J. R. Liu, "SER performance analysis and optimum power allocation for decode-and-forward cooperation protocol in wireless networks," in Proc. IEEE Wireless Commun. Netw. Conf. (WCNC'05), New Orleans, LA, Mar. 13-17, 2005, vol. 2, pp. 984-989.

[8] A. K. Sadek, W. Su, and K. J. R. Liu, "Performance analysis for multinode decode-and-forward relaying in collaborative wireless networks," in Proc. IEEE Int. Conf. Acoust., Speech, Signal Process. (ICASSP), Philadelphia, PA, Mar. 19-23, 2005, vol. 3, pp. 521-524.

[9] _ "A class of cooperative communication protocols for multi-node wireless networks," in Proc. IEEE Int. Workshop Signal Process. Adv. Wireless Commun. (SPAWC), New York, NY, Jun. 2005, pp. 560-564.

[10] T. E. Hunter and A. Nosratinia, "Cooperation diversity through coding," in Proc. IEEE Int. Symp. Inf. Theory, Lausanna, Switzerland, Jul. 2002, p. 220.

[11] J. Boyer, D. D. Falconer, and H. Yanikomeroglu, "Multihop diversity in wireless relaying channels," IEEE Trans. Commun., vol. 52, no. 10, pp. $1820-1830$, Oct. 2004

[12] M. K. Simon and M.-S. Alouini, "A unified approach to the performance analysis of digital communication over generalized fading channels," Proc. IEEE, vol. 86, no. 9, pp. 1860-1877, Sep. 1998. 
[13] J. W. Craig, "A new, simple and exact result for calculating the probability of error for two-dimensional signal constellations," in Proc. IEEE Milcom., Boston, MA, 1991, pp. 25.5.1-25.5.5.

[14] G. Kramer, M. Gastpar, and P. Gupta, "Cooperative strategies and capacity theorems for relay networks," IEEE Trans. Inf. Theory, vol. 51, no. 9, pp. 3037-3063, Sep. 2005.

[15] G. Scutari and S. Barbarossa, "Distributed space-time coding for regenerative relay networks," IEEE Trans. Wireless Commun., vol. 4, no. 5, pp. 2387-2399, Sep. 2005.

[16] S. Barbarossa, Multiantenna Wireless Communication Systems. Norwood, MA: Artech House, 2005.

[17] L. Zheng and D. N. C. Tse, "Diversity and multiplexing: A fundamental tradeoff in multiple-antenna channels," IEEE Trans. Inf. Theory, vol. 49, no. 5, pp. 1073-1096, May 2003.

[18] H. S. Wang and P.-C. Chang, "On verifying the first-order Markovian assumption for a Rayleigh fading channel model," IEEE Trans. Veh. Technol., vol. 45, no. 2, pp. 353-357, Mar. 1996.

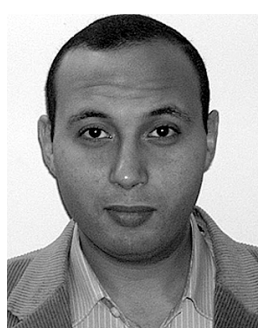

Ahmed K. Sadek (S'04) received the B.S. (with highest honors) and M.S. degrees in electrical engineering from Alexandria University, Alexandria, Egypt, in 2000 and 2003, respectively, and he is currently working towards the Ph.D. degree at the Department of Electrical and Computer Engineering, University of Maryland, College Park.

His current research interests are in the areas of cooperative communications, wireless and sensor networks, MIMO/orthogonal frequency-division multiplexing (OFDM) systems, and blind signal

processing techniques.

Mr. Sadek received a Graduate School Fellowship from University of Maryland in 2003 and 2004, and his B.S. graduation project won the first award in the IEEE Egypt Section Undergraduate Student Contest in 2000.

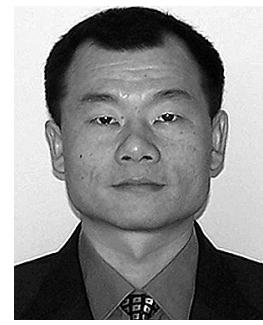

Weifeng Su (M'03) received the B.S. and Ph.D. degrees in mathematics from Nankai University, Tianjin, China, in 1994 and 1999, respectively, and the Ph.D. degree in electrical engineering from the University of Delaware, Newark, in 2002.

$\mathrm{He}$ is an Assistant Professor at the Department of Electrical Engineering, State University of New York (SUNY), Buffalo. From June 2002 to March 2005, he was a Postdoctoral Research Associate with the Department of Electrical and Computer Engineering and the Institute for Systems Research (ISR), University of Maryland, College Park. His research interests span a broad range of areas from signal processing to wireless communications and networking, including space-time coding and modulation for MIMO wireless communications, MIMO-OFDM systems, cooperative communications for wireless networks, and ultrawideband (UWB) communications.

Dr. Su received the Signal Processing and Communications Faculty Award from the University of Delaware in 2002 as an outstanding graduate student in the field of signal processing and communications. In 2005, he received the Invention of the Year Award from the University of Maryland. He serves as an Associate Editor for the IEEE TRANSACTIONS ON VEHICULAR TECHNOLOGY.

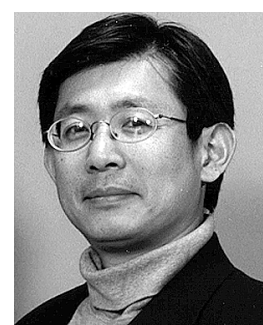

K. J. Ray Liu (F'03) received the B.S. degree from the National Taiwan University, Taipei, Taiwan, R.O.C., in 1983, and the Ph.D. degree from the University of California at Los Angeles (UCLA), Los Angeles, in 1990, both in electrical engineering.

$\mathrm{He}$ is a Professor, Associate Chair, and Director of Graduate Studies and Research of Electrical and Computer Engineering Department, University of Maryland, College Park. His research contributions encompass broad aspects of wireless communications and networking, information forensics and security, multimedia communications and signal processing, bioinformatics and biomedical imaging, and signal processing algorithms and architectures.

Dr. Liu is the recipient of numerous honors and awards including Best Paper Awards from the IEEE Signal Processing Society (twice), the IEEE Vehicular Technology Society, and The European Association for Signal and Image Processing (EURASIP), the IEEE Signal Processing Society Distinguished Lecturer, the EURASIP Meritorious Service Award, and the National Science Foundation Young Investigator Award. He also received the Poole and Kent Company Senior Faculty Teaching Award and the Invention of the Year Award, both from University of Maryland. He is a Vice President-Publications and on the Board of Governors of IEEE Signal Processing Society. He was the Editor-in-Chief of the IEEE SIGNAL PROCESSING MAGAZINE and the founding Editor-in-Chief of the EURASIP Journal on Applied Signal Processing. 